\title{
Methodology for Establishing Segmentation Strategy for Large Metal Components from Nuclear Power Plants with Consideration of Packaging into Containers
}

\author{
Jae Min Lee $\mathbb{D}$, Jae Hak Cheong $\mathbb{D}$, and Jooho Whang $\mathbb{B}$ \\ Department of Nuclear Engineering, Kyung Hee University, 1732 Deogyeong-daero, Giheung-gu, Yongin-si, Gyeonggi-do 17104, \\ Republic of Korea \\ Correspondence should be addressed to Jae Hak Cheong; jhcheong@khu.ac.kr
}

Received 15 June 2020; Accepted 30 March 2021; Published 16 April 2021

Academic Editor: Arkady Serikov

Copyright (c) 2021 Jae Min Lee et al. This is an open access article distributed under the Creative Commons Attribution License, which permits unrestricted use, distribution, and reproduction in any medium, provided the original work is properly cited.

A methodology for segmenting large metal components from nuclear power plants has been developed with a view to minimizing the number of containers to emplace segmented pieces. Spherocylinder-type and rectangular prism-type objects are modeled in shapes, and equations to calculate heights, widths, lengths, or angles for segmentation and the number of pieces are derived using geometric theorems, with a hypothetical 'virtual rectangle' being introduced for simplification. Applicability of the new methodology is verified through case studies assuming that each segmented piece is packaged into a $200 \mathrm{~L}$ container, and a procedure for adjusting potential overestimation of the segmented pieces due to the virtual rectangle is proposed. The new approach results in fewer segmented pieces but more containers than an existing segmentation study using 3D modeling. It is demonstrated that the number of containers can be further reduced, however, if the generalized methodology is followed by $3 \mathrm{D}$ modeling. In addition, it is confirmed that the generalized approach is also applicable to a nonstandard shapes such as ellipsoidal shape but only under limited conditions. Sensitivity analyses are conducted by changing dimensions of the objects and container, which brings about an optimal dimension of container as well. The generalized approach would be utilized either alone in decommissioning planning to estimate waste from segmentation of large metal components or combined with 3D modeling to optimize segmentation operation.

\section{Introduction}

The International Atomic Energy Agency (IAEA) estimates that about 6200 tons of radioactive waste is to be generated from decommissioning of a unit of 900-1300 MWe pressurized water reactor (PWR), and approximately $67 \%$ of the waste is in a form of either surface-contaminated or neutron-activated metal [1]. Large metal components such as reactor pressure vessel (RPV), steam generator (SG), and pressurizer (PRZ) are generated from decommissioning of PWRs as highly activated or contaminated metal waste, which have been disposed of in either of the following ways: (i) segmentation and disposal of the segmented pieces, (ii) direct disposal of one-piece component with or without decontamination $[2,3]$. Though the feasibility of each disposal option may vary on a case-by-case basis, the segmentation and disposal option has been reported to be beneficial to reduce the occupational exposure and the cost for radioactive waste management through cutting off the highly activated or contaminated part from the less contaminated part [4].

If a large metal component is decided to be segmented, a segmentation strategy, which can be defined as a process to find a set of lengths and/or angles to segment an object with a view to optimizing subsequent management of resulting segmented pieces, should be considered early in planning for decommissioning. It has been reported that the segmentation strategy can be driven by various factors such as the type of waste container, disposal cost, and transportation requirements [5].

Various experiences in segmentation of large metal components at nuclear power plants (NPPs) under 
decommissioning can be found in literature. For instance, the segmentation strategy using 3D modeling software (e.g., CAD-CAM), the segmentation techniques adopted for large components, and the results have been reported in various literature works including decommissioning experience reports for actual NPPs and a few technical papers [4, 6-10]. However, these past studies have presented neither generalized nor detailed methodologies to establish the segmentation strategy.

Relatively recently, a few investigators have attempted to calculate angles or lengths for segmentation based upon the geometric shapes of large metal components using $3 \mathrm{D}$ modeling software in order to establish the segmentation strategy. In 2013, Lee et al. have suggested an approach to segmenting the cylindrical part of an RPV at an angle derived using a 3D digital model based upon two constraints on dimensions (i.e., width and depth) of segmented pieces being packaged into cuboid-shape containers [11]. However, the study neither presented any logical segmentation strategy for the hemispherical bottom part of the RPV nor considered the height of segmented pieces as a constraint. Furthermore, the segmentation result of the study could not be verified since the exact dimensions of the target RPV were not specified. The approach proposed in the above study would not be directly used for a general application due to the above limitations. Choi et al. have proposed a different approach to establishing a strategy to segment both hemispherical and cylindrical parts of the RPV of Kori NPP Unit 1 under the constraint of the height of segmented pieces equal to or less than the height of containers in 2016 [12]. They have virtually segmented the RPV using a 3D design software SOLIDWORKS ${ }^{\circledR}$ and showed the segmentation results with exact dimensions of the RPV and the segmentation angles and heights of the resulting pieces, which enables reproduction of their work. However, the approach would not be generally applied for segmentation planning since no rationale to select the segmentation angles has been clearly addressed.

Based on the above literature review, this study aims at proposing a new generalized and geometrical approach to optimal segmentation of representative types of large metal components from NPPs with consideration of the dimensions of both the large metal component and the container in which the segmented pieces are to be emplaced. Applicability of the generalized approach is to be verified through comparison with comparable techniques; however, the method proposed by Lee et al. (2013) [11] has limitations to comparison as indicated above. Therefore, the method proposed by Choi et al. (2016) [12] is used to verify the generalized approach for the same object and storage container. Besides, the effect of various factors on the efficiency of segmentation strategy (e.g., the number of segmented pieces) is to be analyzed through sensitivity analysis.

\section{Methodology}

2.1. Classification of Large Metal Components and Basic Assumptions. Though various types of large metal components are generated from NPPs, the representative shapes of them are firstly categorized into the followings: (i) a spherocylinder-type object such as RPV, SG, and PRZ and (ii) a rectangular prism-type object representing walls of spent fuel storage rack. It is assumed that the spherocylinder-type object simply consists of a cylindrical part and two hemispherical parts with the same thickness, while the rectangular prism-type object is assumed to be in a hollow cuboid shape with a constant thickness without lid or bottom. The shapes of the spherocylinder-type and rectangular prism-type objects are schematically shown in Figure 1 together with symbols for dimensions.

Even for the same object of large metal component, the derived segmentation strategy would be widely varied depending upon the volume and/or the shape of containers to be used. In this study, a cylindrical storage container widely used at NPPs in operation or under decommissioning is assumed to be used to emplace the segmented pieces as shown in Figure 2. Furthermore, in order to generalize the methodology for establishing the segmentation strategy, the possible preliminary cutting of nonradioactive parts is excluded, and just one segmented piece is assumed to be loaded into a single container.

Segmentation of the object is not needed, and just direct packaging of one-piece object without cutting is practical enough under the following conditions:

(i) Spherocylinder-type object: $2 \cdot\left(r_{C}+t_{C}\right)<d_{D}$ and $\left\{h_{C}+2 \cdot\left(r_{H}+t_{H}\right)\right\}<h_{D}$

(ii) Rectangular-prism-type object: $\sqrt{w_{R}^{2}+l_{R}^{2}}<d_{D}$ and $h_{R}<h_{D}$

2.2. Segmentation of Spherocylinder-Type Object. The cylindrical part and the two hemispherical parts of a spherocylinder-type object (see Figure 1(a)) can be segmented horizontally and vertically, and then the resulting pieces can be emplaced into the container as depicted in Figures 2 and 3.

2.2.1. Segmentation of Cylindrical Part. When the cylindrical part of a spherocylinder-type object is segmented in horizontal direction, the height of segmented pieces should be lower than the reference value (i.e., the height of container, $\left.h_{D}\right)$. It is noted that the thickness of the cylindrical part $\left(t_{C}\right)$ should be at least shorter than the diameter of container $\left(d_{D}\right)$ as a basic constraint for segmentation. If the cylindrical part is segmented horizontally to the same reference height $h_{D}$, the height of the last segmented piece is to be the same as or lower than that of the preceding segmented pieces. In this study, therefore, the uniform height of segmented pieces from horizontal cutting $\left(h_{C, Z, S}^{\prime}\right)$ of the cylindrical part is calculated by dividing the height of the cylindrical part $\left(h_{C}\right)$ by the number of pieces from horizontal segmentation $\left(N_{C, Z, S}\right)$ in order to give rise to the minimum number of horizontal cuttings as 


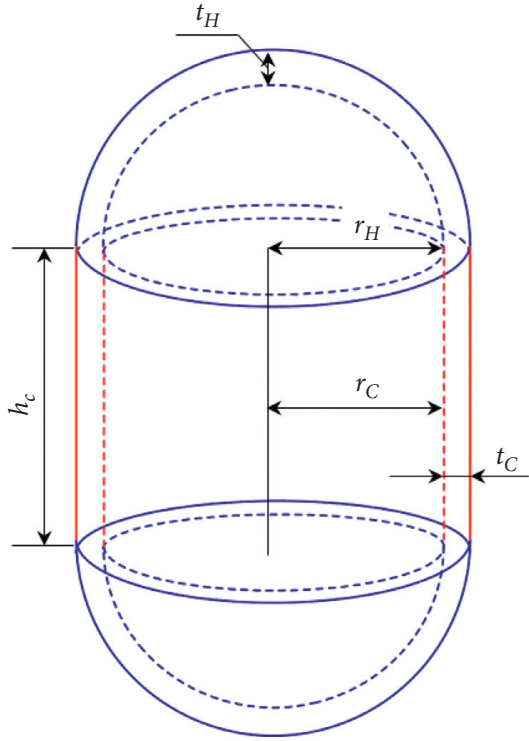

(a)

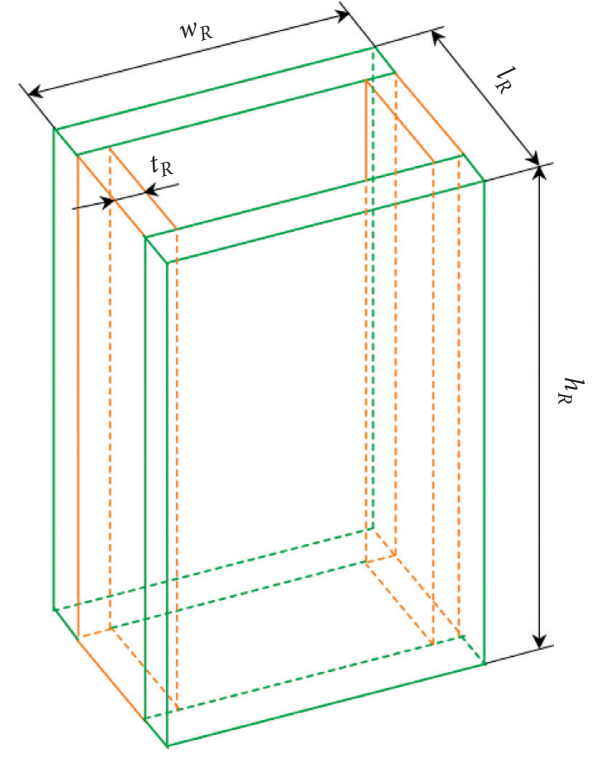

(b)

Figure 1: Schematic view of two types of objects representing large metal components: (a) spherocylinder-type object; (b) rectangular prism-type object.

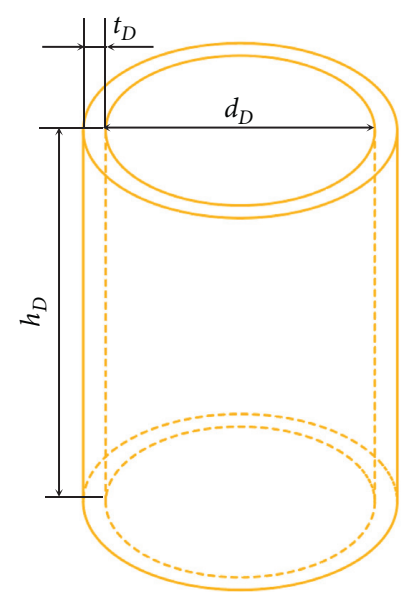

FIgURE 2: Schematic view of a cylindrical storage container into which segmented pieces are to be emplaced.

$$
h_{C, Z, S}^{\prime}=\frac{h_{C}}{N_{C, Z, S}}= \begin{cases}\frac{h_{C}}{\left(h_{C} / h_{C, Z, S}\right)}, & \left(\text { If } \frac{h_{C}}{h_{C, Z, S}} \text { is an integer }\right), \\ \frac{h_{C}}{\operatorname{Int}\left(h_{C} / h_{C, Z, S}\right)+1}, & \left(\text { If } \frac{h_{C}}{h_{C, Z, S}} \text { is not an integer }\right) .\end{cases}
$$

After being horizontally segmented to the uniform height $h_{C, Z, S}^{\prime}$ as per (1), the cylindrical part should be further segmented vertically and the top view of resulting pieces would be in a shape of partial annulus (see Figures 3 and 4). In order to emplace the vertically segmented piece into the container, the chord length of the piece $\left(a_{C, V, S}\right)$ should be less than the reference value (i.e., the inner diameter of container, $d_{D}$ ), which can be calculated using a well-known chord length formula as follows [13]:

$$
a_{C, V, S}=2 \cdot\left(r_{C}+t_{C}\right) \cdot \sin \left(\frac{\theta_{C, V, S}}{2}\right)<d_{D},
$$

which is a necessary condition rather than a sufficient condition for the resulting piece to be packaged into the container, however, since a certain thick piece (i.e., $t_{C}$ is high) may not be fit into the container even if its chord length does not exceed the container's inner diameter (i.e., $\left.a_{C, V, S}<d_{D}\right)$. Though $t_{C}$ assumed not to exceed $d_{D}$ in horizontal segmentation should be further constrained for 


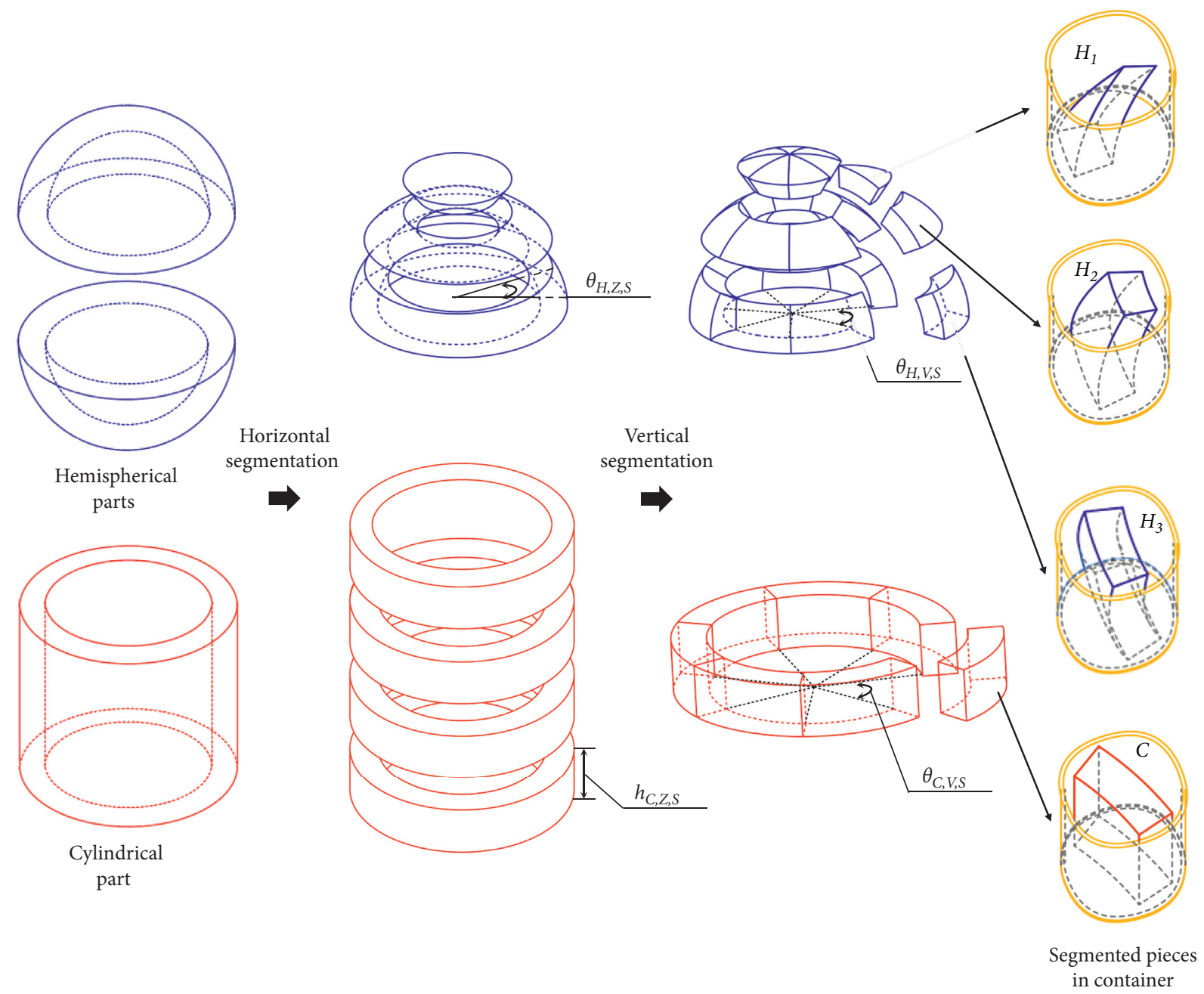

Figure 3: Schematic view of sequential segmentation strategy for the spherocylinder-type object shown in Figure 1(a).

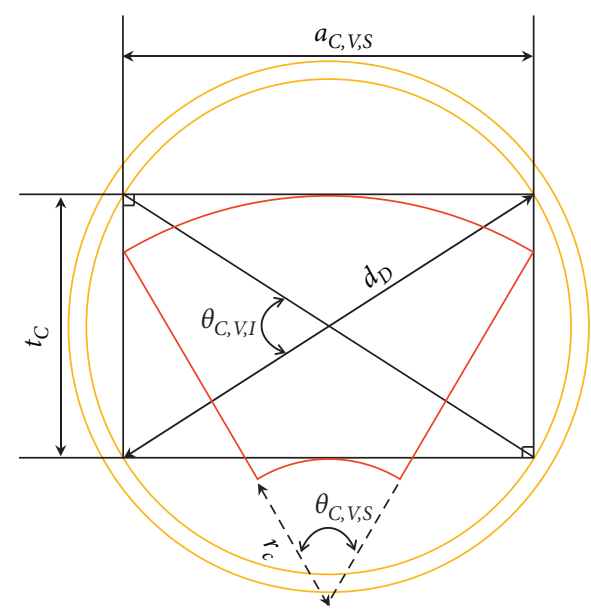

FIGURE 4: Top view of the piece resulting from horizontal and subsequent vertical segmentations of the cylindrical part of a spherocylindertype object emplaced into a container together with a virtual rectangle assumed in this study.

vertical segmentation, it seems to be complicated to derive a general constraint (e.g., an inequality) using geometrical parameters of the segmented piece. In this study, therefore, a hypothetical 'virtual rectangle' inscribing the inner circle of the container with the length of $t_{C}$, whose center of the upper side meets with the apex of the partial annulus of the resulting piece, has been assumed for simplification as represented in Figure 4. 
Once the virtual rectangle is introduced as in Figure 4, $a_{C, V, S}$ can be equated with the included angle of the virtual rectangle $\left(\theta_{C, V, I}\right)$ and the inner diameter of the container $\left(d_{D}\right)$ using the chord length formula as

$$
a_{C, V, S}=d_{D} \cdot \sin \left(\frac{180^{\circ}-\theta_{C, V, I}}{2}\right) \text {. }
$$

Likewise, $\theta_{C, V, I}$ can be derived as a function of $t_{C}$ and $d_{D}$ based upon the chord length formula as well.

$$
t_{C}=d_{D} \cdot \sin \left(\frac{\theta_{C, V, I}}{2}\right) \text { or } \theta_{C, V, I}=2 \cdot \sin ^{-1}\left(\frac{t_{C}}{d_{D}}\right) \text {. }
$$

Accordingly, $a_{C, V, S}$ can be calculated by (3) and (4) if $t_{C}$ and $d_{D}$ are given.

Based upon the same approach adopted in horizontal segmentation, it is assumed that the horizontally segmented cylindrical part is to be cut in vertical direction into pieces all having the same segmentation angle. Accordingly, the uniform segmentation angle for vertical cutting $\left(\theta_{C, V, S}^{\prime}\right)$ can be determined by dividing $360^{\circ}$ by the number of pieces produced per vertical segmentation $\left(N_{C, V, S}\right)$ as:

$$
\theta_{C, V, S}^{\prime}=\frac{360^{\circ}}{N_{C, V, S}}= \begin{cases}\frac{360^{\circ}}{\left(360^{\circ} / \theta_{C, V, S}\right)}, & \left(\text { If } \frac{360^{\circ}}{\theta_{C, V, S}} \text { is an integer }\right), \\ \frac{360^{\circ}}{\operatorname{Int}\left(360^{\circ} / \theta_{C, V, S}\right)+1}, & \left(\text { If } \frac{360^{\circ}}{\theta_{C, V, S}} \text { is not an integer }\right) .\end{cases}
$$

Finally, the number of segmented pieces to be generated from the cylindrical part $\left(N_{C, S}\right)$ can be calculated as below.

$$
N_{C, S}=N_{C, Z, S} \cdot N_{C, V, S} \text {. }
$$

2.2.2. Segmentation of Hemispherical Parts. When the cylindrical part of a spherocylinder-type object is segmented in horizontal direction as depicted in Figure 3, the top or bottom hemispherical part can be segmented horizontally at angle $\theta_{H, Z, S}$ and the resulting segmented piece can be further vertically segmented at angle $\theta_{H, V, S}$. In order to estimate $\theta_{H, Z, S}\left(\leq 45^{\circ}\right.$ for one or more segmentations) making the segmented piece fit into the container to the maximum, the chord length of the finally segmented piece $\left(a_{H, Z, S}\right)$ shown in Figure 5 can be equated with the reference value $h_{D}$, which is the height of container where the finally segmented piece is to be emplaced, using the chord length formula as follows.

$$
a_{H, Z, S}=2 \cdot\left(r_{H}+t_{H}\right) \cdot \sin \left(\frac{\theta_{H, Z, S}}{2}\right)<h_{D} \text { or } \theta_{H, Z, S}<2 \cdot \sin ^{-1}\left(\frac{h_{D}}{2 \cdot\left(r_{H}+t_{H}\right)}\right) .
$$

The hemispherical part is also assumed to be segmented into pieces at the same horizontal angle and with the same chord length. Therefore, the uniform segmentation angle for horizontal cutting $\left(\theta_{H, Z, S}^{\prime}\right)$ of the hemispherical part can be calculated by dividing $90^{\circ}$ by the number of pieces from horizontal segmentation $\left(N_{H, Z, S}\right)$ as

$$
\theta_{H, Z, S}^{\prime}=\frac{90^{\circ}}{N_{H, Z, S}}= \begin{cases}\frac{90^{\circ}}{\left(90^{\circ} / \theta_{H, Z, S}\right)}, & \left(\text { If } \frac{90^{\circ}}{\theta_{H, Z, S}} \text { is an integer }\right), \\ \frac{90^{\circ}}{\operatorname{Int}\left(90^{\circ} / \theta_{H, Z, S}\right)+1}, & \left(\text { If } \frac{90^{\circ}}{\theta_{H, Z, S}} \text { is not an integer }\right) .\end{cases}
$$

After horizontal segmentation of the hemispherical part at the uniform angle $\theta_{H, Z, S}^{\prime}$, the horizontally segmented piece should be subsequently segmented to the vertical direction. The resulting segmented piece would be in a shape of the volume element of a spherical shell (see Figures 3 and 6).
As shown in Figure 6, the vertical segmentation angle $\left(\theta_{H, V, S}\right)$ should be derived in order to decide the dimension of the finally segmented piece which is to be fit into the inner space of the given container as much as possible. Therefore, the depth of segmented piece $\left(w_{H, S}\right)$ as schematically defined in 


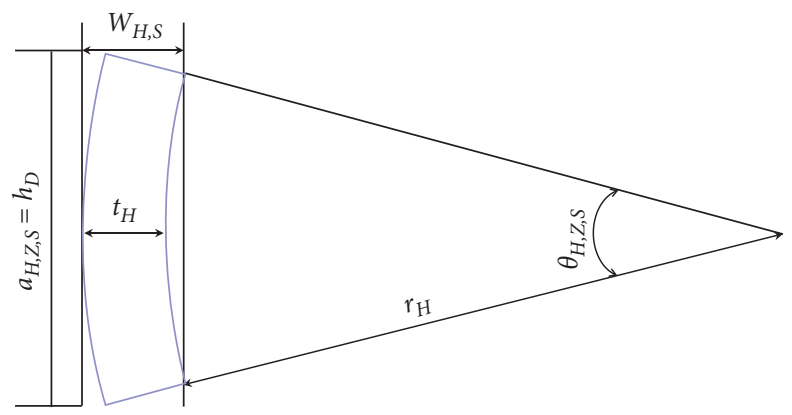

FIGURE 5: Side view of the piece resulting from horizontal and subsequent vertical segmentations of a hemispherical part of a spherocylinder-type object.

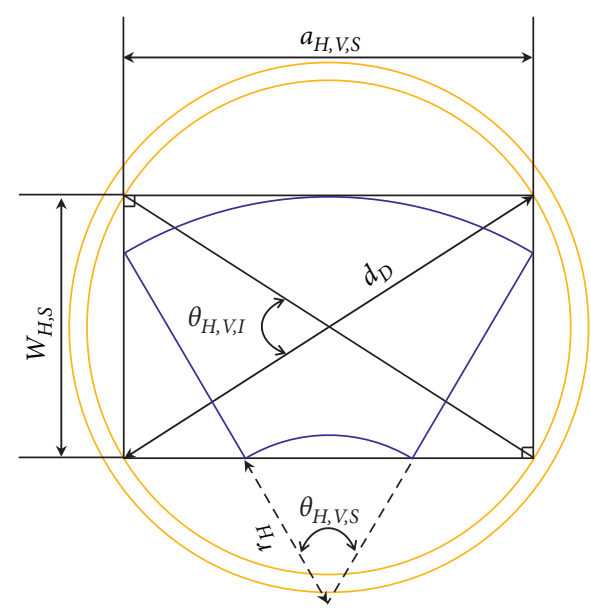

FIGURE 6: Top view of the piece resulting from horizontal and subsequent vertical segmentations of a hemispherical part of spherocylindertype object emplaced into a container together with the virtual rectangle assumed in this study.

Figure 5 should be additionally determined in segmentation of the hemispherical parts. In order to estimate $w_{H, S}$ making the segmented piece fit into the container to the maximum, the virtual rectangle with length $a_{H, V, S}$ and depth $w_{H, S}$ enclosing the maximum cross section of the final segmented piece is assumed again in this study. The virtual rectangle is to inscribe the inner circle of the container as shown in Figure 6. The length of the virtual rectangle $a_{H, V, S}$ (or the vertical segmentation angle $\theta_{H, V, S}$ ) can be calculated using (9) which is derived in the same way as $a_{C, V, S}$ in (3) and (4).

$$
a_{H, V, S}=d_{D} \cdot \sin \left(\frac{180^{\circ}-2 \cdot \sin ^{-1}\left(w_{H, S} / d_{D}\right)}{2}\right) \text { or } \theta_{H, V, S}=2 \sin ^{-1}\left(\frac{a_{H, V, S}}{2\left(r_{H}+t_{H}\right)}\right) \text {. }
$$

It is noted that (9) implies $w_{H, S}<d_{D}$, since the independent variable of arcsine function should not be greater than " 1 " (see Figures 5 and 6). Besides, the width of the virtual rectangle $w_{H, S}$ can be calculated using the chord length formula as well (see Figure 6).

$$
w_{H, S}=t_{H}+r_{H}-r_{H} \cdot \cos \left(\frac{\theta_{H, Z, S}^{\prime}}{2}\right) \text {. }
$$

On the other hand, the uniform angle for vertical cutting $\left(\theta_{H, V, S}\right)$ of the hemispherical part can be calculated by dividing $360^{\circ}$ by the number of resulting pieces from a vertical segmentation $\left(N_{H, V, S}\right)$ as follows.

$$
\theta_{H, V, S}^{\prime}=\frac{360^{\circ}}{N_{H, V, S}}= \begin{cases}\frac{360^{\circ}}{\left(360^{\circ} / \theta_{H, V, S}\right)}, & \left(\text { If } \frac{360^{\circ}}{\theta_{H, V, S}} \text { is an integer }\right), \\ \frac{360^{\circ}}{\operatorname{Int}\left(360^{\circ} / \theta_{H, V, S}\right)+1}, & \left(\text { If } \frac{360^{\circ}}{\theta_{H, V, S}} \text { is not an integer }\right) .\end{cases}
$$


Therefore, the number of segmented pieces $\left(N_{H, S}\right)$ from the two hemispherical parts can be calculated by

$$
N_{H, S}=2 \cdot\left(N_{H, Z, S} \cdot N_{H, V, S}\right) .
$$

Finally, the total number of segmented pieces from the spherocylinder-type object $\left(N_{\mathrm{SC}, S}\right)$ can be given by adding (6) and (12) as follows.

$$
N_{\mathrm{SC}, S}=N_{C, S}+N_{H, S}
$$

2.3. Segmentation of Rectangular Prism-Type Object. The rectangular prism-type object considered in this study consists of four rectangular walls of the same height and thickness without ceiling or bottom as shown in Figure 1(b). It is basically assumed that the thickness $\left(t_{R}\right)$ of the object is lower than the container's inner diameter $\left(d_{D}\right)$. As depicted in Figure 7, both front and back walls are separated in advance from side walls (i.e., left and right walls) prior to horizontal segmentation. Accordingly, the length $\left(l_{R}-2 t_{R}\right)$ of left or right wall, once the front and back walls have been segmented, is lower than the original length $\left(l_{R}\right)$ by twice the wall thickness $\left(t_{R}\right)$.

The rectangular prism-type object whose four walls have been separated is to be further segmented horizontally at height $h_{R, Z, S}$ which is shorter than the reference height $h_{D}$. The uniform height $\left(h_{R, Z, S}^{\prime}\right)$ of all segmented pieces in a cuboid shape resulting from subsequent horizontal cutting can be calculated from the height of walls $\left(h_{R}\right)$ and the number of horizontal segmentation operations for each wall $\left(N_{R, Z, S}\right)$ as

$$
\begin{aligned}
& w_{\mathrm{FB}, V, S}^{\prime}=\frac{w_{R}}{N_{\mathrm{FB}, V, S}}= \begin{cases}\frac{w_{R}}{\left(w_{R} / w_{\mathrm{FB}, V, S}\right)}, & \left(\text { If } \frac{w_{R}}{w_{\mathrm{FB}, V, S}} \text { is an integer }\right), \\
\frac{w_{R}}{\operatorname{Int}\left(w_{R} / w_{\mathrm{FB}, V, S}\right)+1}, & \left(\text { If } \frac{w_{R}}{w_{\mathrm{FB}, V, S}} \text { is not an integer }\right),\end{cases} \\
& l_{\mathrm{LR}, V, S}^{\prime}=\frac{l_{R}-2 t_{R}}{N_{\mathrm{LR}, V, S}}= \begin{cases}\frac{l_{R}-2 t_{R}}{\left(\left(l_{R}-2 t_{R}\right) / l_{\mathrm{LR}, V, S}\right)}, & \left(\text { If } \frac{l_{R}-2 t_{R}}{l_{\mathrm{LR}, V, S}} \text { is an integer }\right), \\
\frac{l_{R}-2 t_{R}}{\operatorname{Int}\left(\left(l_{R}-2 t_{R}\right) / l_{\mathrm{LR}, V, S}\right)+1}, & \left(\text { If } \frac{l_{R}-2 t_{R}}{l_{\mathrm{LR}, V, S}} \text { is not an integer }\right) .\end{cases}
\end{aligned}
$$

The total number of final pieces from the rectangular prism-type object $\left(N_{R, S}\right)$ is to be given as

$$
N_{R, S}=2 \cdot\left(N_{\mathrm{FB}, V, S}+N_{\mathrm{LR}, V, S}\right) \cdot N_{R, Z, S}
$$

2.4. Overall Procedure for Implementing Generalized Segmentation Approach. Based upon a set of descriptions and equations in Sections 2.2 to 2.3, the whole procedure for

$$
h_{R, Z, S}^{\prime}=\frac{h_{R}}{N_{R, Z, S}}= \begin{cases}\frac{h_{R}}{\left(h_{R} / h_{R, Z, S}\right)}, & \left(\text { If } \frac{h_{R}}{h_{R, Z, S}} \text { is an integer }\right), \\ \frac{h_{R}}{\operatorname{Int}\left(h_{R} / h_{R, Z, S}\right)+1}, & \text { (If } \left.\frac{h_{R}}{h_{R, Z, S}} \text { is not an integer }\right) .\end{cases}
$$

The horizontally segmented pieces are to be subsequently segmented in vertical direction and then emplaced into the cylindrical container (see Figure 2). The configuration of the storage container in which the segmented pieces from the rectangular prism-type object are packaged is presented in Figure 8.

In Figure 8, the vertical segmentation width and length $\left(w_{\mathrm{FB}, V, S}, l_{\mathrm{LR}, V, S}\right)$ can be calculated using the Pythagorean theorem or the chord length formula as adopted in deriving equation (9) as

$$
w_{\mathrm{FB}, V, S}=l_{\mathrm{LR}, V, S}=\sqrt{d_{D}^{2}-t_{R}^{2}}=d_{D} \cdot \sin \left(\frac{180-2 \sin ^{-1}\left(t_{R} / d_{D}\right)}{2}\right) .
$$

As shown in Figure 7, the width and length of resulting pieces from horizontal and subsequent vertical segmentations of front/back walls and side walls are given in $w_{F B, V, S}$ and $l_{L R, V, S}$, respectively. Accordingly, the uniform width and length of the finally segmented pieces $\left(w_{F B, V, S}^{\prime}\right.$ and $l_{L R, V, S}^{\prime}$, respectively) can be derived from the width and length of two types of walls $\left(w_{R}\right.$ and $l_{R}-2 t_{R}$, respectively) and the number of pieces from vertical segmentations $\left(N_{\mathrm{FB}, V, S}\right.$ and $N_{\text {LR,V,S}}$, respectively) as

establishing a segmentation strategy for large metal components can be summarized as shown in Figure 9.

\section{Results and Discussion}

\subsection{Case Study I: Segmentation Strategy for Spherocylinder-} Type Object

3.1.1. General Segmentation Approach. The generalized approach to establishing segmentation strategy proposed in 


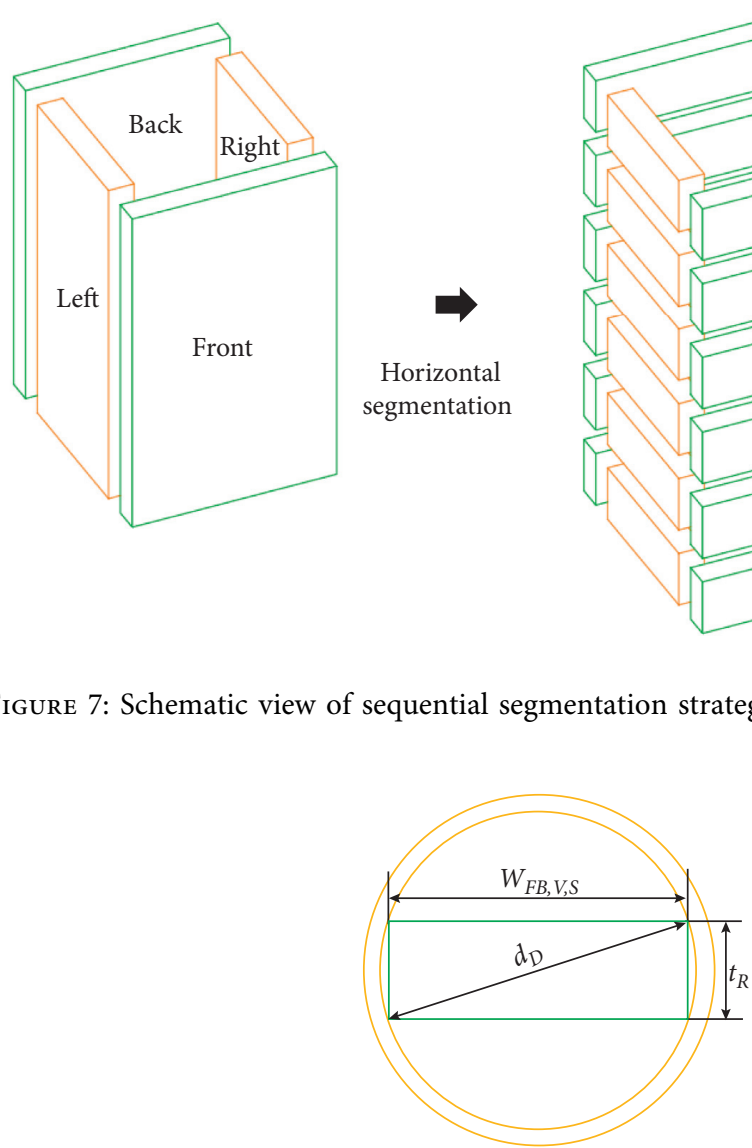

(a)

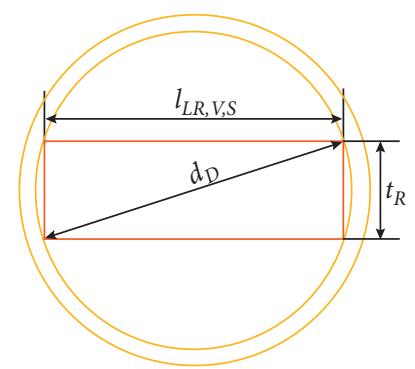

(b)

Figure 8: Top view of a finally segmented piece from the rectangular prism-type object placed into the container: (a) a segmented piece from front/back walls; (b) a segmented piece from left/right walls.

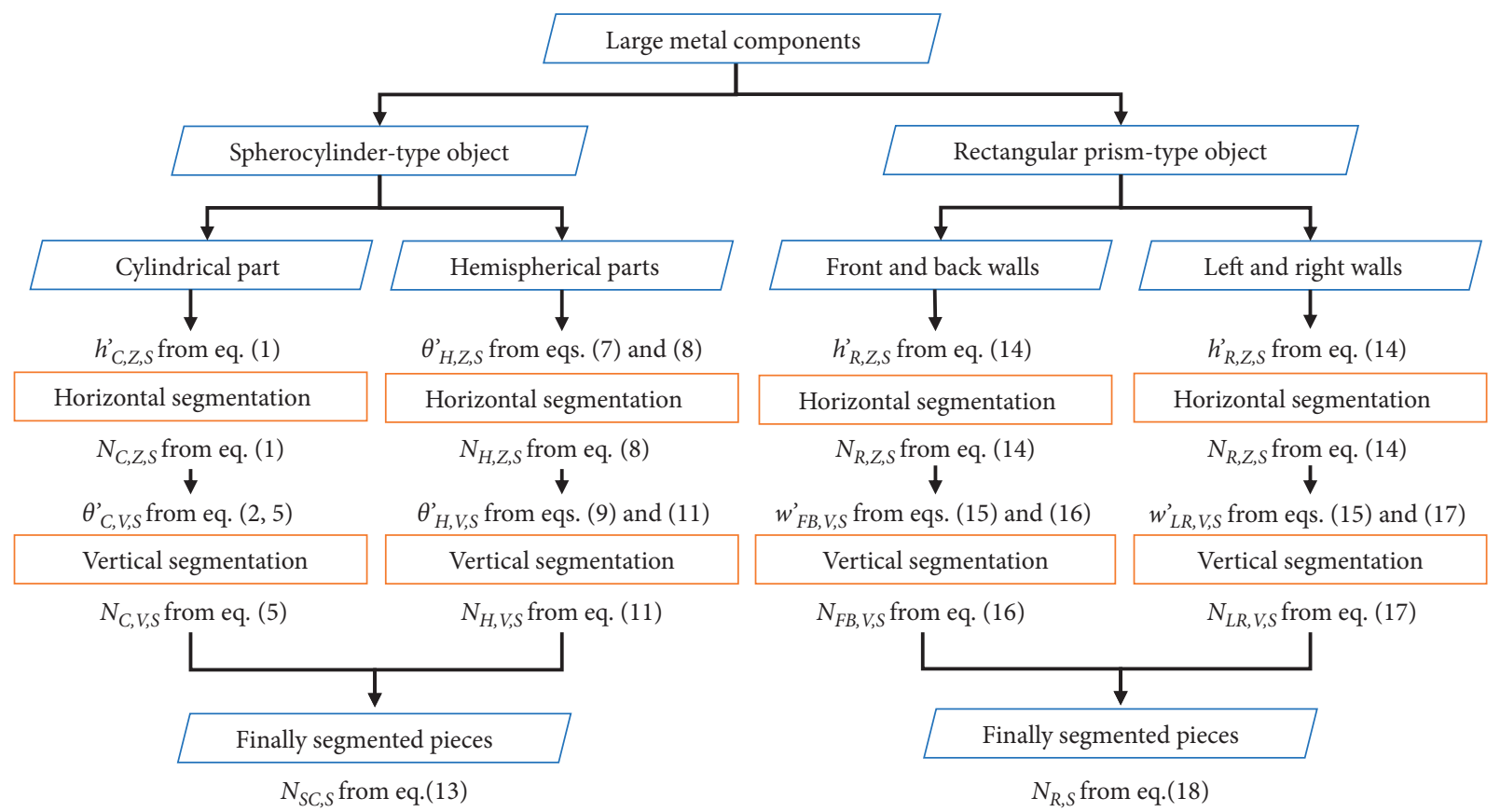

FIGURE 9: Flow chart of generalized approach to establishing segmentation strategy for large metal components developed in this study. 
this study (see Figure 9) has been implemented to a spherocylinder-type object of specified dimensions in order to demonstrate its applicability. The geometry and dimensions of the spherocylinder-type object $\left(r_{C}=1.5 \mathrm{~m}\right.$, $t_{C}=0.2 \mathrm{~m}, h_{C}=7.6 \mathrm{~m}, t_{H}=0.2 \mathrm{~m}$, and $\left.r_{H}=1.5 \mathrm{~m}\right)$ are assumed by referring to the typical size of RPVs with simplification as appropriate. In addition, those of the container $\left(d_{D}=0.567 \mathrm{~m}, h_{D}=0.884 \mathrm{~m}\right)$ are assumed to be the same as $200 \mathrm{~L}$ steel drum which has been most commonly used for storage of low- and intermediate-level radioactive waste (LILW) at NPPs in Korea [14].

The uniform segmentation height $\left(h_{C, Z, S}\right)$ and segmentation angle $\left(\theta_{C, V, S}\right)$ have been derived by inserting the specified dimension values $\left(r_{C}, t_{C}, h_{C}, t_{H}\right.$, and $\left.r_{H}\right)$ into (1), (4), and (5) for the cylindrical part. Likewise, the uniform segmentation angles $\left(\theta_{H, Z, S}, \theta_{H, V, S}\right)$ for the hemispherical parts have been calculated using (7) to (9) and (11) together with the given dimension values as above. Using the derived segmentation height and angles for segmentation strategy shown in Table 1, the number of segmented pieces for each type of segmentation $\left(N_{C, Z, S}, N_{C, V, S}, N_{H, Z, S}\right.$, and $\left.N_{H, V, S}\right)$ has been calculated from (1), (5), (8), and (11). Furthermore, the number of segmented pieces $\left(N_{C, S}=189\right)$ from the cylindrical part is calculated by multiplying $N_{C, Z, S}(=9)$ by $N_{C, V, S}(=21)$ and that $\left(N_{H, S}=126\right)$ from the hemispherical parts is given by multiplying $N_{H, Z, S}(=3)$ by $N_{H, V, S}(=21)$ and then doubled for the two (top and bottom) parts, in accordance with (6) and (12). As summarized in Table 1, the total number of final pieces $\left(N_{S C, S}\right)$ produced from segmentation of the specified spherocylinder-type object turns out to be 315 .

\subsubsection{Adjustment of Errors due to Virtual Rectangle.}

Potential gaps between the number of segmented pieces generally derived in Column (A) of Table 1 and the actual minimum number of pieces would be induced by the virtual rectangles assumed in this study (see Figures 4 and 6). Accordingly, a methodology for adjusting the gap, if any, is additionally proposed. For that purpose, the top view of a finally segmented piece from the cylindrical part of a spherocylinder-type object without the virtual rectangle is generally shown in Figure 10, which is comparable to Figure 4.

As shown in Figure 10, the chord length of the segmented piece $\left(a_{C, V, S}^{\prime \prime}=\overline{A B}\right)$ should not exceed the inner diameter of the container $\left(d_{D}=2 \cdot \overline{O Q}\right)$ as

$$
a_{C, V, S}^{\prime \prime}=2 \cdot\left(r_{C}+t_{C}\right) \cdot \sin \left(\frac{\theta_{C, V, S}^{\prime \prime}}{2}\right)<d_{D} .
$$

$\alpha$ and $\beta$ in Figure 10 can be represented as general equations using the chord length formula for $\overline{C D}$ and the Pythagorean theorem for $\triangle O R B$.

$$
\begin{aligned}
& \alpha=r_{C} \cdot \sin \left(\frac{\theta_{C, V, S}^{\prime \prime}}{2}\right), \\
& \beta=\left[\left(\frac{d_{D}}{2}\right)^{2}-\delta^{2}\right]^{(1 / 2)}=\left[\left(\frac{d_{D}}{2}\right)^{2}-(\varepsilon-\gamma)^{2}\right]^{(1 / 2)} .
\end{aligned}
$$

Points $\mathrm{C}$ and $\mathrm{D}$ should be within the inner area of the container (i.e., $\overline{F C}<\overline{F C^{\prime}}$ or $\alpha<\beta$ ) for the segmented piece to be emplaced into the container. Therefore, (20) and (21) can be rewritten using the Pythagorean theorem for $\triangle O F C^{\prime}$ and the definition of cosine for $\triangle A A^{\prime} D$ as below.

$$
r_{C} \cdot \sin \left(\frac{\theta_{C, V, S} \prime}{2}\right)<\left[\left(\frac{d_{D}}{2}\right)^{2}-\left\{t_{C} \cdot \cos \left(\frac{\theta_{C, V, S}^{\prime \prime}}{2}\right)-\left\{\left(\frac{d_{D}}{2}\right)^{2}-\left(\frac{a_{C, V, S}{ }^{\prime \prime}}{2}\right)^{2}\right\}^{(1 / 2)}\right\}^{2}\right.
$$

That is, (19) and (22) can be considered as general constraints for the segmented piece to be emplaced into the container if the virtual rectangle is not assumed.

In order to adjust the possible gap which may be caused by the virtual rectangle assumed in the generalized segmentation approach of this study, the range of new segmentation angle $\left(\theta_{C, V, S}{ }^{\prime \prime}\right)$ is calculated by solving (19) and (22) through a numerical analysis for the cylindrical part as given in Section 3.1 (i.e., $t_{C}=0.2 \mathrm{~m}, r_{C}=1.5 \mathrm{~m}$, and $\left.d_{D}=0.567 \mathrm{~m}\right)$. If the resulting possible range of the segmentation angle $\left(0^{\circ}<\theta_{C, V, S}<18.8^{\circ}\right)$ is inserted into (5), the number of horizontally segmented pieces turns out to be a natural number in the range of $N_{C, V, S} \geq 20$, which implies that the maximum segmentation angle and the minimum number of segmentation pieces are to be $18^{\circ}$ and 20 , respectively. As shown in Table 1, the gap in the number of segmented pieces from horizontal segmentation due to the virtual rectangle turns out to be "1" (i.e., $N_{C, V, S}=21$ versus $N_{C, V, S}^{\prime}=20$ ).

Likewise, it is shown in Table 1 that the segmentation angle $\left(\theta_{H, V, S}=17.1^{\circ}\right)$ and the number of segmented pieces $\left(N_{H, V, S}=21\right)$ from vertical segmentation of the hemispherical part can be adjusted to $\theta_{H, V, S}^{\prime \prime}=18^{\circ}$ and $N_{H, V, S}^{\prime}=20$, respectively, by applying the equivalent constraints of (19) and (22) to the respective case after replacing $t_{C}\left(=t_{H}\right)$ with $w_{H, S}$ (see Figure 6).

Though the gap in the number of vertically segmented pieces between the general approach and the adjustment is "1" (i.e., $\Delta N_{C, V, S}=N_{C, V, S^{\prime}}-N_{C, V, S}^{\prime}=1$ ) for the reference case (e.g., $t_{C}=0.2 \mathrm{~m}, d_{D}=0.567 \mathrm{~m}$, and $\left(t_{C} / d_{D}\right)=35.3 \%$ ) assumed in this study, it may be varied with difference in dimensional values of the object. It turns out to that the gap $\Delta N_{C, V, S}$ increases with increasing $t_{C}$ while other dimensional values are fixed, through application of the adjustment 
TABLE 1: Segmentation strategy derived for the spherocylinder-type object assumed in this study and comparison with the results from 3D modeling using Rhino [15].

\begin{tabular}{|c|c|c|c|c|c|c|}
\hline \multirow{3}{*}{ Target } & \multirow{3}{*}{ Parameter } & \multirow{3}{*}{ Equation } & \multicolumn{3}{|c|}{ Results } & \multirow{3}{*}{$\begin{array}{l}\text { Relative error of general approach } \\
\qquad((A)-(C) /(C)) \times 100\end{array}$} \\
\hline & & & \multicolumn{2}{|c|}{ This study } & \multirow{2}{*}{$\begin{array}{l}\text { 3D modeling } \\
(\mathrm{C})\end{array}$} & \\
\hline & & & $\begin{array}{l}\text { General approach } \\
\text { (A) }\end{array}$ & Adjustment (B) & & \\
\hline \multirow{5}{*}{ Cylindrical part } & $h_{C, Z, S}^{\prime}$ & (1) & $0.844 \mathrm{~m}$ & $0.844 \mathrm{~m}$ & $0.844 \mathrm{~m}$ & - \\
\hline & $\theta_{C, V, S}$ & (5) & $17.1^{\circ}$ & $18^{\circ}$ & $18^{\circ}$ & $-4.78 \%$ \\
\hline & $N_{C, Z, S}$ & (1) & 9 & 9 & 9 & - \\
\hline & $N_{C, V, S}$ & (5) & 21 & 20 & 20 & $+5.00 \%$ \\
\hline & $N_{C, S}$ & (6) & 189 & 180 & 180 & $+5.00 \%$ \\
\hline \multirow{5}{*}{$\begin{array}{l}\text { Hemispherical } \\
\text { parts }\end{array}$} & $\theta_{H, Z, S}^{\prime}$ & (8) & $30^{\circ}$ & $30^{\circ}$ & $30^{\circ}$ & - \\
\hline & $\theta_{H, V, S}$ & (11) & $17.1^{\circ}$ & $18^{\circ}$ & $18^{\circ}$ & $-4.78 \%$ \\
\hline & $N_{H, Z, S}$ & (8) & 3 & 3 & 3 & - \\
\hline & $N_{H, V, S}$ & (11) & 21 & 20 & 20 & $+5.00 \%$ \\
\hline & $N_{H, S}$ & $(12)$ & 126 & 120 & 120 & $+5.00 \%$ \\
\hline Total & $N_{S C, S}$ & $(13)$ & 315 & 300 & 300 & $+5.00 \%$ \\
\hline
\end{tabular}

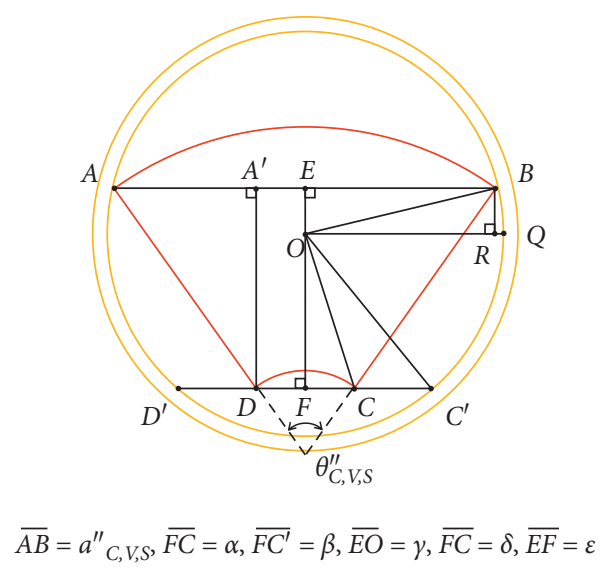

Figure 10: General top view of a finally segmented piece from the cylindrical part of a spherocylinder-type object depicted for deriving adjustment algorithm for errors induced by the virtual rectangle.

procedure to multiple cases. For instance, $\Delta N_{C, V, S}$ increases to " 3 " and " 5 " if $t_{C}$ increases to $0.4 \mathrm{~m}\left(\left(t_{C} / d_{D}\right)=70.5 \%\right)$ and $0.5 \mathrm{~m}\left(\left(t_{C} / d_{D}\right)=88.2 \%\right)$, respectively. However, it is noted that the thickness of representative large metal components from NPPs is usually reported to be comparable to the reference case assumed in this study: RPV of APR 1000 $\left(t_{C}=0.205 \mathrm{~m}, d_{D}=0.567 \mathrm{~m},\left(t_{C} / d_{D}\right)=36 \%\right)$, SG of Turkey Point NPP $\left(t_{C}=0.08 \mathrm{~m}, d_{D}=0.567 \mathrm{~m},\left(t_{C} / d_{D}\right)=14 \%\right)$, and so forth $[16,17]$. It can be said that, therefore, the general approach developed in this study can be practically applied without significant error from the adjusted result.

3.1.3. Verification Using 3D Modeling. In order to evaluate the relative efficiency of the generalized segmentation approach and the adjustment methodology proposed in this study, the same spherocylinder-type object has been virtually segmented using the well-known 3D modeling software Rhinoceros ${ }^{\circledR}$ (i.e., Rhino) in a manual way under the same constraints addressed in Section 2.2 as well, and the three results are given in Table 1 . The general segmentation strategy derived in this study shows $+5 \%$ less efficiency than the manual 3D modeling in terms of the total number of final segmented pieces to be produced (i.e., 315 versus 300 from the two approaches, respectively). The small difference in the two segmentation strategies is induced from the difference in the estimated segmentation angles, while the segmentation height proposed from the two methods is the same. The difference in the estimated segmentation angles has come from the virtual rectangles assumed as depicted in Figures 4 and 6 .

In addition, the result from $3 \mathrm{D}$ modeling turns out to be the same as the adjusted result in terms of segmentation height, angles, and resulting number of pieces (see Table 1). This implies that the strategy established by the general segmentation approach (see Section 3.1.1) can be adjusted by the proposed methodology (see Section 3.1.2) not relying on additional manual 3D modeling.

3.2. Case Study II: Segmentation Strategy for Rectangular Prism-Type Object. The generalized segmentation approach developed has been implemented to a rectangular prismtype object of specified dimensions $\left(w_{R}=10 \mathrm{~m}, l_{R}=10 \mathrm{~m}\right.$, 
$h_{R}=8 \mathrm{~m}$, and $\left.t_{R}=0.2 \mathrm{~m}\right)$ as well, while the segmented pieces have been assumed to be packaged into a $200 \mathrm{~L}$ container as given in Section 3.1.1.

The uniform segmentation height $\left(h_{R, Z, S}\right)$, width $\left(w_{F B, V, S}{ }^{\prime}\right)$, and length $\left.\left(l_{L R, V, S}\right)^{\prime}\right)$ have been derived by inserting the specified dimensional values $\left(w_{R}, l_{R}, h_{R}\right.$, and $\left.t_{R}\right)$ of the object into (14) to (17). Using the derived segmentation height, length, and width for segmentation strategy shown in Table 2, the number of segmented pieces for each type of segmentation $\left(N_{R, Z, S}, N_{\mathrm{FB}, V, S}\right.$, and $\left.N_{\mathrm{LR}, V, S}\right)$ has been calculated from (14), (16), and (17). Furthermore, the number of segmented pieces $\left(N_{\mathrm{FB}, S}=380\right)$ from the front and back walls is calculated by multiplying $N_{R, Z, S}(=10)$ by $N_{\mathrm{FB}, V, S}(=$ 19 ) and then doubled for the two target walls. Likewise, the number of segmented pieces $\left(N_{\mathrm{LR}, S}=380\right)$ from the left and right walls can be calculated. As given in Table 2, the total number of final pieces $\left(N_{R, S}\right)$ produced from segmentation of the specified rectangular prism-type object turns out to be 760 .

The same rectangular prism-type object has been virtually segmented using the 3D modeling software Rhino, under the same constraints given in Section 2.3, in order to test the relative efficiency of the segmentation strategy generally derived in this study. As shown in Table 2, the segmentation strategy derived in this study shows the same efficiency (i.e., no relative error) as the manual 3D modeling in terms of the total number of final segmented pieces to be produced (i.e., 760 total pieces).

Additional consideration of potential adjustment of segmentation height, width, and length or the number of segmented pieces resulting from the general segmentation approach as above is not needed, since the virtual rectangle has not been assumed in the segmentation of the rectangular prism-type object (see Figure 8).

\subsection{Comparison with an Existing Study on Establishment of} Segmentation Strategy. The generalized approach proposed in this study is additionally compared with a comparable technique reported in the past study on segmentation of a large metal component from an NPP in which geometries and dimensions of the target component and container are specified [12]. The geometries and dimensions of the target large metal component to be segmented (i.e., the RPV of Kori NPP Unit 1) and the container (i.e., M II-10T MOSAIK ${ }^{\circledR}$ cask used in Stade NPP in Germany) are assumed to be the same as those considered in the past study whose specifications are given in Table 3 .

The procedure for deriving the segmentation strategy for the spherocylinder-type object adopted in Section 3.1 is applied to the target object and container as given in Table 3 . As a result, a segmentation strategy producing 182 segmented pieces is derived in this study, while the past study reported the number of resulting segmented pieces to be 504 in total (see Table 4). It is noted that the number of segmented pieces is just $36.1 \%$ of that reported by the past study.

However, the number of containers (i.e., 126) emplacing segmented pieces reported in the past study is much smaller than that of the resulting pieces (i.e., 504) [12]. The difference is caused by the approach to emplacement of multiple segmented pieces into a container using 3D modeling software (SOLIDWORKS ${ }^{\circledR}$ ) adopted in the past study, while this study assumes just one single segmented piece to be emplaced into a container (see Figure 3).

In order to compare the number of resulting containers from the two studies under the same conditions, therefore, we tried to reduce the total number of containers by assuming that multiple pieces derived from the general approach can be emplaced into a container. The volume of each piece in Type $\mathrm{C}$ is the same as or higher than any other pieces in Types $\mathrm{H}_{1}$ to $\mathrm{H}_{3}$ (see Figure 3), since the segmented piece from the cylindrical part has a horizontal curvature only while those from the hemispherical parts have curvatures in horizontal and vertical directions as well. It is noted that additional cutting of the smaller segmented pieces is more feasible than that of larger ones in terms of segmentation area.

As an initial trial, therefore, 84 segmented pieces of three types (i.e., Types $\mathrm{H}_{1}$ to $\mathrm{H}_{3}$ in Figure 3 ) from the hemispherical parts derived using the general approach are assumed to be segmented further vertically in half, and then 168 smaller pieces of new types (i.e., Types $H_{1}^{\prime}$ to $H_{3}^{\prime}$ ) are produced. However, 98 segmented pieces from the cylindrical part (i.e., Type $\mathrm{C}$ in Figure 3 ) are not assumed to be cut further. As a result, the total number of pieces is to increase from $182\left(N_{C, S}=98\right.$ and $\left.N_{H, S}=84\right)$ to $266\left(N_{C, S}=98\right.$ and $\left.N_{H, S}=168\right)$. It is demonstrated by $3 \mathrm{D}$ modeling using Rhino that 84 sets of combinations of two types of additionally segmented pieces (i.e., total 168 pieces in either of the following combinations: $H_{1}^{\prime}-H_{2}^{\prime}, H_{1}^{\prime}-H_{3}^{\prime}$, and $H_{2}^{\prime}-H_{3}^{\prime}$ ) can be emplaced into the same number of 84 containers already storing a Type $\mathrm{C}$ piece each, while the remaining 14 containers emplace just a single segmented Type C piece.

It is noted that the resulting number of containers may decrease by $54 \%$ (from 182 to 98 containers), which is smaller than that from the past study, using the generalized segmentation strategy and subsequent additional segmentation supported by $3 \mathrm{D}$ modeling.

3.4. Case Study III: Segmentation Strategy for Ellipsoidal Shape. The generalized approach to establishing segmentation strategy proposed in this study can be comprehensively applied to the 'cylindrical part of spherocylinder-type' and 'rectangular prism-type' objects of any components or structures. If the top or bottom head of RPV is not perfectly hemispherical, however, the methodology in this study may not be fully applicable. In order to show the applicability of the new methodology for nonhemispherical head of pressure vessel, the generalized approach to establishing segmentation strategy proposed in this study has been implemented to the bottom head of VVER-1000 reactor whose shape is reported to be 'ellipsoidal' in accordance with the categories of head shapes in the American Society of Mechanical Engineers (ASME) Boiler and Pressure Vessel Code (BPVC) Section VIII Division 1 [18]. It was assumed that the shape of the bottom head of VVER-1000 reactor is of hypothetical 
TABLE 2: Segmentation strategy derived for the specified rectangular prism-type object assumed in this study and comparison with the results from 3D modeling using Rhino [15].

\begin{tabular}{|c|c|c|c|c|c|c|}
\hline \multirow{3}{*}{ Target } & \multirow{3}{*}{ Parameter } & \multirow{3}{*}{ Equation } & \multicolumn{3}{|c|}{ Results } & \multirow{3}{*}{$\begin{array}{l}\text { Relative error of general approach } \\
\qquad((A)-(C) /(C)) \times 100\end{array}$} \\
\hline & & & \multicolumn{2}{|c|}{ This study } & \multirow{2}{*}{$\begin{array}{l}\text { 3D modeling } \\
(\mathrm{C})\end{array}$} & \\
\hline & & & $\begin{array}{c}\text { General approach } \\
\text { (A) }\end{array}$ & Adjustment (B) & & \\
\hline \multirow{4}{*}{$\begin{array}{l}\text { Front and back } \\
\text { walls }\end{array}$} & $h_{R, Z, S}^{\prime}$ & (14) & $0.8 \mathrm{~m}$ & N/A & $0.8 \mathrm{~m}$ & - \\
\hline & $w_{F B, V, S}^{\prime, ~}$ & (16) & $0.52 \mathrm{~m}$ & N/A & $0.52 \mathrm{~m}$ & - \\
\hline & $N_{R, Z, S}$ & (14) & 10 & N/A & 10 & - \\
\hline & $N_{\mathrm{FB}, V, S}$ & $(16)$ & 19 & N/A & 19 & - \\
\hline \multirow{4}{*}{ Left and right walls } & $h_{R, Z, S}^{\prime}$ & (14) & $0.8 \mathrm{~m}$ & N/A & $0.8 \mathrm{~m}$ & - \\
\hline & $l_{\mathrm{LR}, V, S}^{\prime}$ & (17) & $0.50 \mathrm{~m}$ & N/A & $0.50 \mathrm{~m}$ & - \\
\hline & $N_{R, Z, S}$ & (14) & 10 & N/A & 10 & - \\
\hline & $N_{\mathrm{LR}, V, S}$ & $(17)$ & 19 & N/A & 19 & - \\
\hline Total & $N_{R, S}$ & $(18)$ & 760 & N/A & 760 & - \\
\hline
\end{tabular}

TABLE 3: Geometries and dimensions of the RPV and storage container assumed for comparison with the past study [12].

\begin{tabular}{lccc}
\hline Target & Part & Parameter & Value $(\mathrm{m})$ \\
\hline & & $h_{C}$ & 8.24 \\
RPV & Cylindrical part & $r_{C}$ & 1.68 \\
& & $t_{C}$ & 0.17 \\
& Hemispherical parts & $r_{H}$ & 1.68 \\
\multirow{2}{*}{ M II-10T MOSAIK ${ }^{\circledR}$ cask } & $h_{D}$ & $t_{H}$ & 0.17 \\
\hline
\end{tabular}

TABLE 4: Comparison of segmentation strategies derived in this study and reported in the past study for the same target object and container defined in Table 3.

\begin{tabular}{|c|c|c|c|c|c|c|}
\hline \multirow{3}{*}{ Target } & \multirow{3}{*}{ Parameter } & \multirow{3}{*}{ Equation } & \multicolumn{3}{|c|}{ Results } & \multirow{3}{*}{$\begin{array}{l}\text { Relative error of this study with respect to past } \\
\text { study }((A)-(C) /(C)) \times 100\end{array}$} \\
\hline & & & \multicolumn{2}{|c|}{ This study } & \multirow[b]{2}{*}{$\begin{array}{l}\text { Past study } \\
\quad[12]\end{array}$} & \\
\hline & & & $\begin{array}{c}\text { General } \\
\text { approach }(\mathrm{A})\end{array}$ & $\begin{array}{l}\text { Adjustment } \\
\text { (B) }\end{array}$ & & \\
\hline \multirow{5}{*}{ Cylindrical part } & $h_{C, Z, S}^{\prime}$ & (1) & $1.18 \mathrm{~m}$ & $1.18 \mathrm{~m}$ & $1.03 \mathrm{~m}$ & $+14.3 \%$ \\
\hline & $\theta_{C, V, S}^{\prime}$ & (5) & $25.7^{\circ}$ & $25.7^{\circ}$ & $10^{\circ}$ & $+157 \%$ \\
\hline & $N_{C, Z, S}$ & (1) & 7 & 7 & 8 & $-12.5 \%$ \\
\hline & $N_{C, V, S}$ & (5) & 14 & 14 & 36 & $-61.1 \%$ \\
\hline & $N_{C, S}$ & (6) & 98 & 98 & 288 & $-66.0 \%$ \\
\hline \multirow{5}{*}{$\begin{array}{l}\text { Hemispherical } \\
\text { parts }\end{array}$} & $\theta_{H, Z, S}^{\prime}$ & (8) & $30^{\circ}$ & $30^{\circ}$ & $30^{\circ}$ & - \\
\hline & $\theta_{H, V, S}^{\prime}$ & (11) & $25.7^{\circ}$ & $25.7^{\circ}$ & $10^{\circ}$ & $+157 \%$ \\
\hline & $N_{H, Z, S}$ & $(8)$ & 3 & 3 & 3 & - \\
\hline & $N_{H, V, S}$ & (11) & 14 & 14 & 36 & $-61.1 \%$ \\
\hline & $N_{H, S}$ & (12) & 84 & 84 & 216 & $-61.1 \%$ \\
\hline \multirow{2}{*}{ Total } & $N_{S C, S}$ & (13) & 182 & 182 & 504 & $-63.9 \%$ \\
\hline & $N_{D}$ & - & 98 & 98 & 126 & $-22.2 \%$ \\
\hline
\end{tabular}

'hemispherical type' rather than actual 'ellipsoidal type' as shown in Figure 11, while the segmented pieces are assumed to be packaged into the same $200 \mathrm{~L}$ container assumed in this study (see Section 3.1.1).

As shown in Table 5, the uniform segmentation angles $\left(\theta_{E, Z, S}, \theta_{E, V, S}\right)$ for the ellipsoidal bottom head have been calculated using (8) to (11) together with the dimension values given in Figure 11.

Using the segmentation angles derived from the generalized approach $\left(\theta_{E, Z, S}\right.$ and $\left.\theta_{E, V, S}{ }^{\prime}\right)$, the number of segmented pieces for each type of segmentation $\left(N_{E, Z, S}\right.$ and $\left.N_{E, V, S}\right)$ has been calculated from (8) and (11), and the total number of segmented pieces $\left(N_{E, S}=100\right)$ has been calculated by multiplying $N_{E, Z, S}$ by $N_{E, V, S}$. It has been also verified that all types of resulting pieces segmented from the ellipsoidal-type bottom head of VVER 1000 RPV with specific dimensions (i.e., $h_{E}=0.8 \mathrm{~m}, r_{H}=1.8 \mathrm{~m}$ as shown in Figure 11) can be emplaced into the containers by 3D modeling using Rhino (see Figure 12). It is worth noting that the average filling ratio of the containers for the actual ellipsoidal-type bottom head decreases compared to that for the hypothetical hemispherical-shape object (i.e., $22.4 \%$ to $13.9 \%$ ) due to the relative differences in dimensions of the segmented pieces form the two objects. Moreover, the 3D modeling shows that the number of vertical 


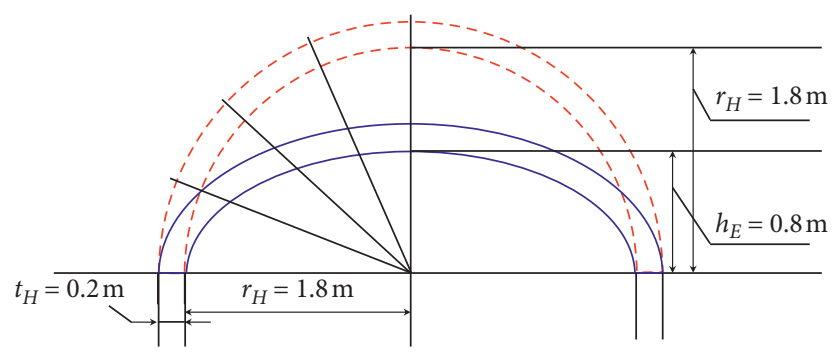

FIGURE 11: Comparison of geometry and dimensions of actual ellipsoidal-type bottom head (blue solid line) of VVER-1000 with hemispherical-type shape assumed in the study (red dotted line).

TABLE 5: Segmentation strategy derived for an ellipsoidal-type bottom head of VVER 1000 RPV and comparison with the results from 3D modeling using Rhino.

\begin{tabular}{|c|c|c|c|c|c|}
\hline \multirow{2}{*}{ Target } & \multirow{2}{*}{ Parameter } & \multirow{2}{*}{ Equation } & \multicolumn{2}{|l|}{ Result } & \multirow{2}{*}{$\begin{array}{l}\text { Relative error of general approach } \\
\qquad((A)-(C) /(C)) \times 100\end{array}$} \\
\hline & & & This study general approach (A) & 3D modeling $(\mathrm{B})$ & \\
\hline \multirow{5}{*}{ Ellipsoidal shape } & $\theta_{E, Z, S}^{\prime}$ & $(8)$ & $22.5^{\circ}$ & $22.5^{\circ}$ & - \\
\hline & $\theta_{E, V, S}$ & (11) & $14.4^{\circ}$ & $15^{\circ}$ & $-4.00 \%$ \\
\hline & $N_{E, Z, S}$ & $(8)$ & 4 & 4 & - \\
\hline & $N_{E, V, S}$ & $(11)$ & 25 & 24 & $+4.17 \%$ \\
\hline & $N_{E, S}$ & $(12)$ & 100 & 96 & $+4.17 \%$ \\
\hline
\end{tabular}
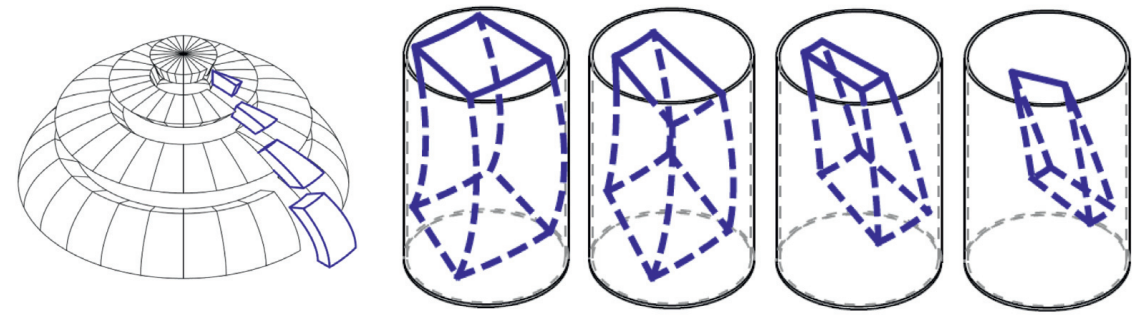

(a)
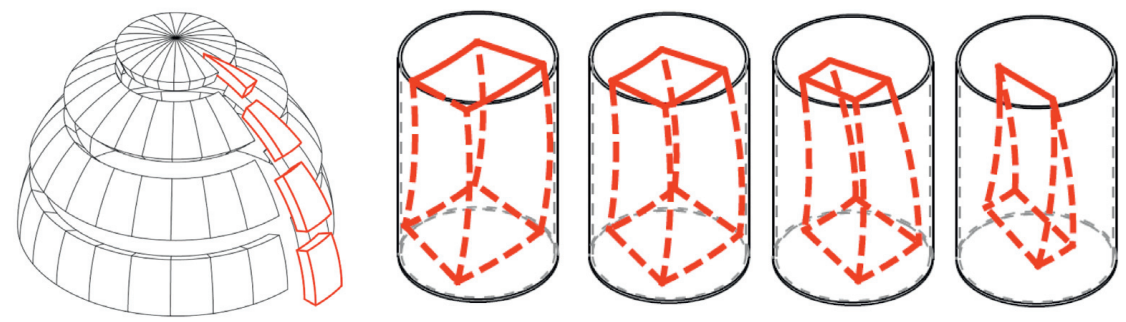

(b)

FIgURE 12: Results of 3D modeling using Rhino for emplacement of all types of segmented pieces into a $200 \mathrm{~L}$ container for (a) actual ellipsoidal-type bottom head of VVER 1000 RPV and (b) hypothetical hemispherical model assumed to apply the generalized approach to the ellipsoidal-type object.

segmentations for the ellipsoidal-type bottom head $\left(N_{E, V, S}\right)$ can be reduced by one (i.e., 25 to 24 ) compared to that for the generalized approach, because the vertical segmentation angle increases from $14.4^{\circ}$ to $15^{\circ}$. It turns out that the largest segmented pieces do not fit in the given container, however, if the height of the ellipsoid $\left(h_{E}\right)$ decreases down to below $0.72 \mathrm{~m}$ while the radius $r_{H}=1.8 \mathrm{~m}$ is kept constant.

3.5. Sensitivity Analysis. A series of sensitivity analyses has been conducted by varying geometry and dimensions of the target objects and container, in order to demonstrate the applicability of the generalized segmentation strategy to various conditions and to quantify the effect of major parameters on the number of segmented pieces.

3.5.1. Volume of Spherocylinder-Type Object. The volume of a spherocylinder-type object $V_{S C}$ (in $\mathrm{m}^{3}$ ) given as below has been varied for sensitivity analysis by changing only one parameter (i.e., the thickness $\left(t_{C}=t_{H}\right)$ in Case $1 \mathrm{~A}$, the height $\left(h_{C}\right)$ in Case $1 \mathrm{~B}$, or the inner radius $\left(r_{C}=r_{H}\right)$ in Case $1 C$ ), while other parameter values have been fixed as defined in Section 3.1.1 for the reference case. 
$V_{S C}=\left(\pi \cdot\left(\left(r_{C}+t_{C}\right)^{2}-r_{C}^{2}\right) \cdot h_{C}\right)+\left(\frac{4}{3} \pi \cdot\left(\left(r_{H}+t_{H}\right)^{3}-r_{H}^{3}\right)\right)$.

As shown in Figure 13, the total number of segmented pieces increases with increasing the volume of the spherocylinder-type object from $10.0 \mathrm{~m}^{3}$ by varying one parameter for each case (i.e., $0.096 \mathrm{~m}<t_{C}\left(=t_{H}\right)<0.538 \mathrm{~m}$, $0.754 \mathrm{~m}<r_{C}\left(=r_{H}\right)<3.61 \mathrm{~m}$, and $1.76 \mathrm{~m}<h_{C}<31.6 \mathrm{~m}$, for Cases $1 \mathrm{~A}, 1 \mathrm{~B}$, and $1 \mathrm{C}$, respectively).

In Case $1 \mathrm{~A} \quad\left(0.096 \mathrm{~m}<t_{C}<0.538 \mathrm{~m} \quad\right.$ or $10.0 \mathrm{~m}^{3}<V_{S C}<65.8 \mathrm{~m}^{3}$ ), the number of segmented pieces rapidly increases with increasing $t_{C}$ and approaches infinity when $V_{S C}$ becomes close to about $65.8 \mathrm{~m}^{3}$ (i.e., $\left.t_{C} \longrightarrow 0.538 \mathrm{~m}\right)$. It is certain that the object can be segmented only if the thickness is lower than the container diameter (i.e., $\left.t_{C}<d \mathrm{D}\right)$, where the chord length $\left(a_{C, V, S}\right)$ has a positive value in (3) and (4). It is noted, however, that Figure 13 shows that the maximum thickness of the object to be segmented is lower than the container diameter. As implied in Figure 3, the height $\left(h_{C, Z, S}\right)$ or angle $\left(\theta_{H, Z, S}\right)$ for horizontal segmentation is constrained by the height of container $\left(h_{D}\right)$; however, it is not affected by the thickness of the object $t_{C}\left(=t_{H}\right)$. Accordingly, the convergence of $t_{C}$ to $0.538 \mathrm{~m}$ may not be ascribed to the horizontal segmentation. For the cylindrical part, furthermore, it can be said that $\theta_{C, V, I}$ and $\theta_{C, V, S}$ converge to $180^{\circ}$ and $0^{\circ}$, respectively, and the number of segmented pieces from vertical cutting $\left(N_{C, V, S}\right)$ increases to infinity, if $t_{C}$ increases approximately to $d_{D}$, as inferred from (4) and Figure 4. Hence, the upper limit of $t_{C}$ observed in Figure 13 (i.e., $0.538 \mathrm{~m}$ ) is not to be originated from vertical segmentation of the cylindrical part.

The constraint $w_{H, S}<d_{D}$ should be applied for the vertical segmentation of a hemispherical part (see Figure 5 and equation (9)), while $t_{C}<d_{\mathrm{D}}$ is applied for the vertical segmentation of the cylindrical part. The depth of the segmented piece $w_{H, S}$ should be larger than the thickness of the object $t_{H}\left(=t_{C}\right)$, if $r_{H}\left(=r_{C}\right)>0$ as implied in (10). The upper limit of $t_{H}$ (i.e., $0.538 \mathrm{~m}$ ) can be obtained by varying $t_{H}$ in (7), (8), and (10) and then finding the limiting value to which $t_{H}$ converges when $w_{H, S}$ approaches its upper limit (i.e., $d_{D}$ ).

In Case $1 \mathrm{~B} \quad\left(0.754 \mathrm{~m}<r_{C}<3.61 \mathrm{~m}\right.$ or $10.0 \mathrm{~m}^{3}<$ $\left.V_{S C}<70.0 \mathrm{~m}^{3}\right)$, the number of segmented pieces steeply increases at a certain inner radius of the object where $N_{H, Z, S}$ increases by one and $N_{H, S}$ increases by $N_{H, V, S}$ in (11). The continually increasing number of segmented pieces with increasing $r_{c}$ can be attributed to the increment of $N_{H, V, S}$ with increasing $r_{c}$ as well (see (9) to (11)).

In Case $1 \mathrm{C} \quad\left(1.76 \mathrm{~m}<h_{C}<31.6 \mathrm{~m} \quad\right.$ or $10.0 \mathrm{~m}^{3}<$ $V_{S C}<70.0 \mathrm{~m}^{3}$ ), the number of segmented pieces increases stepwise with increasing the height of the cylindrical part $\left(h_{C}\right)$. It is noted that the number of pieces from the hemispherical parts is not varied while that from vertical segmentation of the cylindrical part $\left(N_{C, V, S}\right)$ increases by 21 whenever $h_{C}$ becomes a multiple of the container height $\left(h_{D}=0.884 \mathrm{~m}\right)$. When $h_{C}$ is reduced to zero $(0)$, the object becomes a spherical object and the total number of segmented pieces is to be 147 , which can be calculated using (6) by letting $N_{C, S}=0$. Furthermore, the steeper slope of Case
$1 \mathrm{~B}$ over Case $1 \mathrm{C}$ at the same volume of the object in Figure 13 is attributed to the fact that both $N_{C, S}$ and $N_{H, S}$ increase with increasing $r_{C}$ in Case $1 \mathrm{~B}$, while only $N_{C, S}$ increases with increasing $h_{C}$ in Case $1 C$.

When $t_{C}$ becomes close to $d_{D}$, the number of segmented pieces increases to infinity as shown in Case $1 \mathrm{~A}$ and direct application of the general segmentation approach may not be practicable. Accordingly, Case 1D shows the number of segmented pieces assuming that the same spherocylindertype object is circumferentially segmented in half in advance and then general segmentation approach is applied. The plots for Case 1A and Case 1D intersect at $t_{C}=0.485 \mathrm{~m}$ (or $V_{S C}=59.0 \mathrm{~m}^{3}$ ) in Figure 13, which implies that a priori circumferential segmentation is preferable with regard to minimizing the total number of segmented pieces when the object is thicker than $0.485 \mathrm{~m}$.

3.5.2. Volume of Rectangular Prism-Type Object. The volume of a rectangular prism-type object $V_{R}\left(\right.$ in $\left.\mathrm{m}^{3}\right)$ is given as

$$
V_{R}=\left\{\left(w_{R} \cdot l_{R}\right)-\left(w_{R}-2 t_{R}\right) \cdot\left(l_{R}-2 t_{R}\right)\right\} \cdot h_{R} .
$$

Accordingly, $V_{R}$ has been varied for sensitivity analysis by changing only one parameter (i.e., the thickness $\left(t_{R}\right)$ in Case $2 \mathrm{~A}$, the width $\left(w_{R}\right)$ in Case $2 \mathrm{~B}$, or the height $\left(h_{R}\right)$ in Case 2C), while other parameter values defined in Section 3.2 for the reference case have been used. The total number of segmented pieces increases with the volume of the rectangular prism-type object $V_{R}$ from $10 \mathrm{~m}^{3}$ by varying one parameter for each case (i.e., $0.032 \mathrm{~m}<t_{R}<0.567 \mathrm{~m}$, $0.500 \mathrm{~m}<w_{R}<46.7 \mathrm{~m}$, and $1.27 \mathrm{~m}<h_{R}<23.0 \mathrm{~m}$, for Cases 2A, 2B, and 2C, respectively) as shown in Figure 14.

In Case 2A $\quad\left(0.032 \mathrm{~m}<t_{R}<0.567 \mathrm{~m} \quad\right.$ or $10.0 \mathrm{~m}^{3}<$ $\left.V_{R}<171 \mathrm{~m}^{3}\right)$, the number of segmented pieces increases with increasing $t_{R}$ and rapidly increases to the infinity when $V_{R}$ approaches about $171 \mathrm{~m}^{3}$ or $t_{R}$ becomes close to the container diameter $d_{D}(=0.567 \mathrm{~m})$. The upper limit of $t_{R}$ (i.e., $t_{R}<d_{D}$ ) can be foreseen from (15) and the fact that no additional constraints on $t_{R}$ have been assumed in this study.

It is observed, in Case 2B $\left(0.500 \mathrm{~m}<w_{R}<46.7 \mathrm{~m}\right.$ or $\left.32.3 \mathrm{~m}^{3}<V_{R}<180 \mathrm{~m}^{3}\right)$, that the number of segmented pieces continually increases stepwise by 20 (i.e., $2 \cdot\left(N_{R, Z, S}\right)=2 \cdot(10)$ as given in Table 2) whenever $w_{R}$ becomes a multiple of $d_{D}(=0.567 \mathrm{~m})$. In Case $2 \mathrm{C}$ $\left(1.27 \mathrm{~m}<h_{R}<23.0 \mathrm{~m}\right.$ or $\left.10.0 \mathrm{~m}^{3}<V_{R}<180 \mathrm{~m}^{3}\right)$, the number of segmented pieces increases stepwise with increasing the height of the walls $\left(h_{R}\right)$. It is noted that the total number of pieces $\left(N_{R, S}\right)$ increases by 76 (i.e., $2 \cdot\left(N_{F B V, S}+N_{L R, V, S}\right)=$ $2 \cdot(19+19)$ as given in Table 2$)$ whenever $h_{R}$ becomes a multiple of the container height $\left(h_{D}=0.884 \mathrm{~m}\right)$.

The number of segmented pieces increases steeply to the infinity if $t_{R}$ becomes close to $d_{D}$ as shown in Case $2 \mathrm{~A}$, which implies that generalized segmentation approach may not be practicable for the given high range of $t_{R}$. Taking into consideration the limitation, Case 2D shows the number of segmented pieces when the same rectangular prism-type object is segmented in half of its thickness in advance and then general segmentation approach is implemented. The two plots for Case 2A and 2D in Figure 14 intersect at $t_{R}=$ 


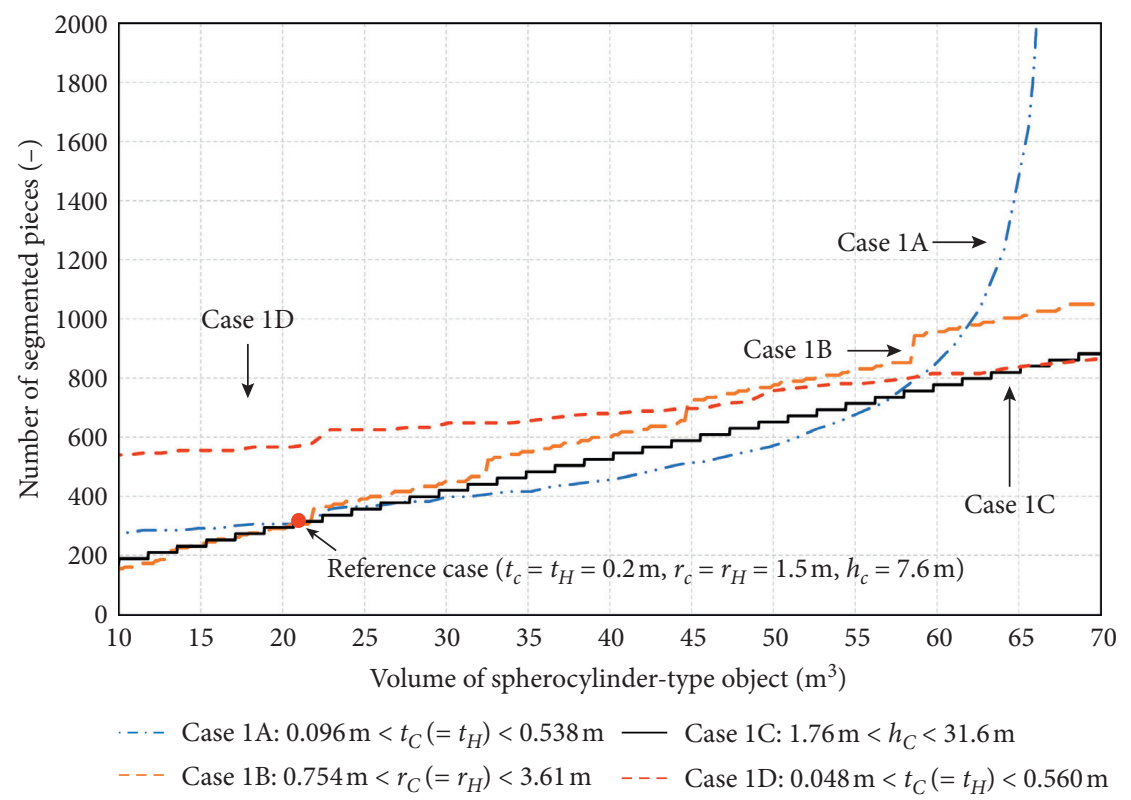

Figure 13: Number of segmented pieces along with variation of the volume of spherocylinder-type object from $10 \mathrm{~m}^{3}$ by changing one parameter only for each case (i.e., thickness in Case 1A, height in Case 1B, and inner radius of object in Case 1C). Case 1D represents a priori circumferential segmentation in half of the same object assumed in Case 1A and application of generalized segmentation approach.

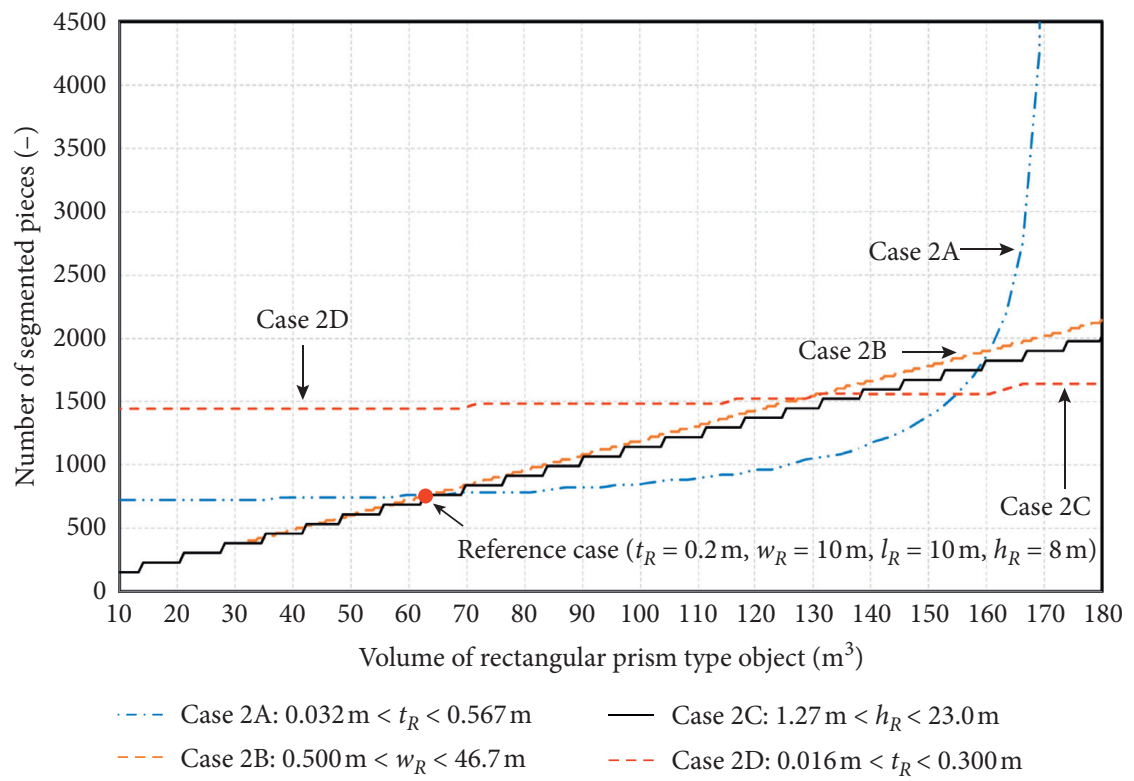

Figure 14: Number of segmented pieces along with variation of the volume of rectangular prism-type object from $10 \mathrm{~m}^{3}$ by changing one parameter only for each case (i.e., thickness in Case 2A, length or width in Case 2B, and height of object in Case 2C). Case 2D represents a priori segmentation in half of the same object assumed in Case $2 \mathrm{~A}$ and application of generalized segmentation approach.

$0.510 \mathrm{~m}$ ( or $V_{R}=154 \mathrm{~m}^{3}$ ), which implies the preference of a priori segmentation of a thick object (i.e., $t_{R}>0.510 \mathrm{~m}$ ) in terms of the total number of segmented pieces.

3.5.3. Height and Radius of $200 \mathrm{~L}$ Container. In order to investigate the effect of variable dimensions of a $200 \mathrm{~L} \mathrm{cy-}$ lindrical container on the number of segmented pieces and determine optimal dimensions of the container, the generalized segmentation strategy has been applied to the spherocylinder- type object for the reference case in Section 3.1.1, while changing the height and radius of the container but keeping the inner volume of the container to be same as the reference case. The volume of steel for all containers is calculated as well by multiplying outer volume of each container by the number of containers (or segmented pieces), for which the thickness of body, lid, and bottom plate of the container is assumed to be $0.0012 \mathrm{~m}$ in accordance with the specifications of cold reduced carbon steel sheets and strip widely used for manufacturing waste storage container [14]. The calculation results (i.e., the 


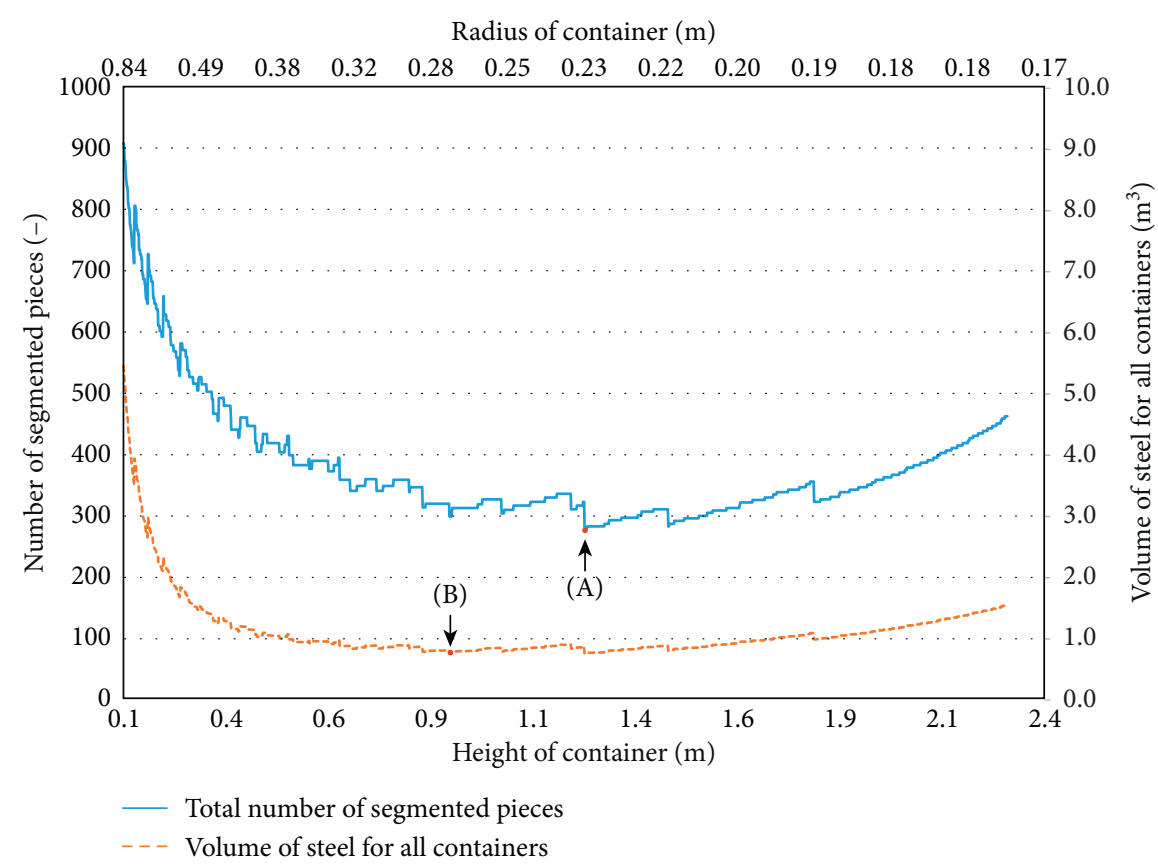

Figure 15: Total number of segmented pieces and volume of steel used for manufacturing all containers with increasing height (or decreasing radius) of $200 \mathrm{~L}$ container. (A) and (B) are the points of the minimum values for the total number of segmented pieces and the volume of steel for all containers, respectively.

number of segmented pieces and the volume of steel for all containers) are shown in Figure 15.

The plot of total number of segmented pieces versus height (or radius) of container shows a U-shape curve in general, which has its minimum 280 pieces at $1.30 \mathrm{~m}$ height (or $0.233 \mathrm{~m}$ radius) of the container. The plot of volume of steel for all containers is convex down at $0.95 \mathrm{~m}$ height (or $0.273 \mathrm{~m}$ radius) of the container, where it shows the minimum volume of $0.759 \mathrm{~m}^{3}$.

All through the range of $h_{D}(\geq 0.100 \mathrm{~m})$ or radius $(\leq 0.842 \mathrm{~m})$ in Figure 15 , the number of horizontally segmented pieces (i.e., $N_{C, Z, S}$ and $N_{H, Z, S}$ ) steeply decreases continuously, which can be inferred from (1), (3), and (5). On the other hand, the number of vertically segmented pieces (i.e., $N_{C, V, S}$ and $N_{H, V, S}$ ) gradually increases within the given range of $h_{D}$ or $d_{D}$. The total number of segmented pieces $\left(N_{S C, S}\right)$ is calculated from products $N_{C, Z, S} \cdot N_{C, V, S}$ and $N_{C, Z, S} \cdot N_{C, V, S}$ (see Table 1); it generally decreases with increasing $h_{D}$ up to around $1.3 \mathrm{~m}$ or decreasing radius down to around $0.233 \mathrm{~m}$ and then generally increases afterwards. The convex down curve shown in Figure 15 and its minimum value of the segmented pieces can be attributed to the analysis as above.

Some irregularities are observed in the general U-shape curve in Figure 15 at certain points of $h_{D}$ (e.g., $\sim 0.249 \mathrm{~m}$ ); that is, $N_{S C, S}$ increases at the specific point while the curve generally shows a decreasing trend in the given range. It has been found that the irregularities are induced when one of the numbers of vertically segmented pieces (e.g., $N_{C, \mathrm{~V}, S}$ for the above point) increases while the other parameters (e.g., $N_{C, Z, S}, N_{H, Z, S}$, and $N_{H, V, S}$ for the above point) are not changed. Meanwhile, the irregularities observed in the range of higher $h_{D}(\geq 1.30 \mathrm{~m})$ can be ascribed to decrement of one of the numbers of horizontally segmented pieces (e.g., $N_{C, Z, S}$ for the point $h_{D} \sim 1.52 \mathrm{~m}$ ), but the other numbers (e.g., $N_{C, \mathrm{~V}, S}, N_{H, V, S}$, and $N_{H, Z, S}$ for the same case) are not changed. The plots of the volume of steel for all containers in Figure 15 can be interpreted in accordance with similar ways as above.

The curves in Figure 15 show that the number of segmented pieces or the volume of steel cannot be calculated when $h_{D}$ exceeds a limiting value $(2.404 \mathrm{~m}$ in this case). It has been found that the depth of vertically segmented pieces from the hemispherical parts becomes higher than the container's inner diameter $\left(w_{H, S}>d_{D}\right)$ when $h_{D}$ exceeds $2.404 \mathrm{~m}$, which implies that the segmented pieces cannot be emplaced into the container (see Figure 6). At the limiting point (i.e., $h_{D}=2.404 \mathrm{~m}$ and $d_{D}=0.344 \mathrm{~m}$ ), $w_{H, S}$ is calculated to be $0.314 \mathrm{~m}$ in (10) and the resulting piece can be packaged into the container. Beyond the limiting point (i.e., $h_{D}>2.404 \mathrm{~m}$ and $\left.d_{D}<0.344 \mathrm{~m}\right), w_{H, S}$ becomes larger than $d_{D}$ (i.e., $w_{H, S}>0.639 \mathrm{~m}$ for the given case), and the segmented piece cannot be emplaced into the container.

On the other hand, the segmented pieces and volume of steel for containers have been also analyzed for the rectangular prism-type object in the reference case defined in Section 3.2. Even if the result is not shown, it generally shows a trend similar to Figure 15 but with different upper limit of $h_{D}(=7.10 \mathrm{~m})$ or $d_{D}(=0.200 \mathrm{~m})$.

\section{Conclusions}

Most of publicly reported practices and studies on segmentation of large metal components from NPPs have focused on introducing the results of $3 \mathrm{D}$ modeling to specific cases without detailed descriptions of methodology and/or technical basis. Based upon a few assumptions for 
simplification, however, generalized equations and procedure for calculating heights, lengths, widths, lengths, or angles for segmentation and the number of resulting pieces using geometric dimensional information of the objects and the container have been derived in this study.

As a result of the case study on the spherocylinder-type object of reference dimension, the generalized approach overestimates the number of segmented pieces $5 \%$ compared to the $3 \mathrm{D}$ modeling conducted for verification. Though the error increases with increasing $\left(t_{C} / d_{\mathrm{D}}\right)$, it is shown that the maximum overestimation of the number of vertically segmented pieces is at most " 2 " within the range of $\left(t_{C} / d_{\mathrm{D}}\right)$ for actual large mental components (i.e., $14-55 \%)$ surveyed in this study. It has been also demonstrated that the overestimation induced from the virtual rectangle can be removed using adjustment algorithm proposed in this study. Besides, it turns out that the segmentation strategy for the rectangular prism-type object can be established as efficiently as the $3 \mathrm{D}$ modeling without adjustment.

Through comparison with an existing study on segmentation of an RPV based upon 3D modeling, the generalized approach gives rise to $36.1 \%$ less number of pieces than the past study (i.e., relative error $-63.9 \%$ ), while the number of containers is overestimated by $44 \%$ which results from an assumption of "one segmented piece to be packaged into a single container" in this study. For comparison under the same condition, the segmented pieces from hemispherical parts have been further vertically cut in half, and then packaging of the total resulting pieces has been tested with a manual 3D modeling, which results in reduction of the need for storage containers (i.e., $77.8 \%$ of the number of containers from past study). In addition, the generalized approach turns out to be also applicable to a nonstandard shapes such as ellipsoidal bottom head of VVER-1000 RPV but only under limited conditions. That is, further study is needed to expand the practical applicability of the new methodology proposed in this study to more various actually installed components and structures at NPPs not belonging to the standard models assumed in this study.

Sensitivity analyses of geometric parameters on the number of pieces derived from general approach to the spherocylinder-type object show linearly increasing curves with local irregularities for $r_{C}\left(=r_{H}\right)$ and $h_{C}$. The upper limit of the object's thickness (i.e., $t_{C}=t_{H}=0.538 \mathrm{~m}$ in this study) for segmentation turns out to be slightly lower than the container's inner diameter $\left(d_{D}=0.567 \mathrm{~m}\right)$, which results from the fact that the depth of segmented piece should be higher than the thickness of the object in segmentation of the hemispherical parts (see Figure 5). Furthermore, it turns out that a priori circumferential cutting of the object would be beneficial if the object's thickness exceeds a limiting value (i.e., $t_{C}=t_{H}>0.485 \mathrm{~m}$ in this study). From sensitivity analyses of the rectangular prism-type object, monotonous increasing of $w_{R}$ and $h_{R}$ with general linearity and local irregularities is observed, but a priori cutting turns out to be considerable if the object is thicker than a certain value (i.e., $t_{R}>0.510 \mathrm{~m}$ in this study). It is noteworthy that the optimal dimensions of $200 \mathrm{~L}$ cylindrical container have been derived as well for two types of objects, through sensitivity analyses in terms of the number of containers and the volume of steel to manufacture all containers, respectively.

It is anticipated that the generalized approach including adjustment algorithm proposed in this study can be used in either of the following ways: (i) standalone application in initial decommissioning planning to establish conceptual segmentation strategy, to estimate the waste from large metal components, and so on; (ii) application in actual segmentation planning to derive angles, heights, etc. for segmentation or to optimize the segmentation strategy (e.g., minimizing the need for containers) prior to or in combination with $3 \mathrm{D}$ modeling.

\section{Abbreviations}

$h_{C}$ : Height of cylindrical part of spherocylinder-type object $(m)$

$h_{R}$ : Height of rectangular prism-type object $(m)$

$h_{D}$ : Inner height of container $(m)$

$h_{C, Z, S}: \quad$ Height of horizontal segmentation of cylindrical part of spherocylinder-type object $(m)$

$h_{R, Z, S}$ : Height of horizontal segmentation of rectangular prism-type object $(m)$

$h_{E}: \quad$ Height of ellipsoidal shape $(m)$

$r_{C}$ : Inner radius of cylindrical part of spherocylindertype object $(m)$

$r_{H}$ : Inner radius of hemispherical part of spherocylinder-type object $(m)$

$t_{C}$ : Thickness of cylindrical part of spherocylindertype object $(m)$

$t_{H}$ : Thickness of hemispherical part of spherocylindertype object $(m)$

$t_{R}: \quad$ Thickness of rectangular prism-type object $(m)$

$d_{D}$ : Inner diameter of container $(m)$

$l_{R}$ : Length of rectangular prism-type object $(m)$

$l_{L R, V, S}: \quad$ Length of vertical segmentation of left and right walls of rectangular prism-type object $(\mathrm{m})$

$a_{C, V, S}: \quad$ Chord length of vertical segmentation of cylindrical part of spherocylinder-type object $(m)$

$a_{H, Z, S}: \quad$ Chord length of horizontal segmentation of hemispherical part of spherocylinder-type object $(m)$

$a_{H, V, S}: \quad$ Chord length of vertical segmentation of hemispherical part of spherocylinder-type object $(m)$

$\theta_{C, V, S}: \quad$ Angle of vertical segmentation of cylindrical part of spherocylinder-type object (degree)

$\theta_{H, Z, S}: \quad$ Angle of horizontal segmentation of hemispherical part of spherocylinder-type object (degree)

$\theta_{H, V, S}: \quad$ Angle of vertical segmentation of hemispherical part of spherocylinder-type object (degree)

$\theta_{E, Z, S}: \quad$ Angle of horizontal segmentation of ellipsoidal shape (degree)

$\theta_{E, V, S}: \quad$ Angle of vertical segmentation of ellipsoidal shape (degree) 
$\theta_{C, V, I}: \quad$ Included angle of virtual rectangle of cylindrical part of spherocylinder-type object (degree)

$\theta_{H, V, I}:$ Included angle of virtual rectangle of hemispherical part of spherocylinder-type object (degree)

$w_{R}: \quad$ Width of rectangular prism-type object $(m)$

$w_{H, S}: \quad$ Depth of segmented piece of hemispherical part of spherocylinder-type object $(m)$

$w_{F B, V, S}$ : Width of vertical segmentation of front and back walls of rectangular prism-type object $(m)$

$N_{C, S}: \quad$ Number of segmented pieces of cylindrical part of spherocylinder-type object (-)

$N_{C, Z, S}$ : Number of horizontally segmented pieces of cylindrical part of spherocylinder-type object (-)

$N_{C, V, S}$ : Number of vertically segmented pieces of cylindrical part of spherocylinder-type object (-)

$N_{H, S}$ : Number of segmented pieces of hemispherical part of spherocylinder-type object (-)

$N_{E, Z, S}$ : Number of horizontally segmented pieces of ellipsoidal shape (-)

$N_{E, V, S}: \quad$ Number of vertically segmented pieces of ellipsoidal shape (-)

$N_{E, S}: \quad$ Number of segmented pieces of ellipsoidal shape $(-)$

$N_{H, Z, S}$ : Number of horizontally segmented pieces of hemispherical part of spherocylinder-type object $(-)$

$N_{H, V, S}:$ Number of vertically segmented pieces of hemispherical part of spherocylinder-type object $(-)$

$N_{R, S}$ : Number of segmented pieces of rectangular prismtype object $(-)$

$N_{F B, V, S}$ : Number of segmented pieces of front and back walls of rectangular prism-type object (-)

$N_{L R, V, S}:$ Number of segmented pieces of left and right walls of rectangular prism-type object (-)

$N_{D}: \quad$ Number of containers (-)

$V_{S C}: \quad$ Volume of spherocylinder-type object $\left(\mathrm{m}^{3}\right)$

$V_{R}: \quad$ Volume of rectangular prism-type object $\left(\mathrm{m}^{3}\right)$

$V_{D}$ : Volume of container $\left(\mathrm{m}^{3}\right)$.

SC: $\quad$ Spherocylinder-type object

FB: $\quad$ Front and back walls

LR: $\quad$ Left and right walls

$R: \quad$ Rectangular prism-type object

C: $\quad$ Cylindrical part of spherocylinder-type object

$H$ : Hemispherical part of spherocylinder-type object

D: $\quad$ Container

$Z$ : Horizontal

$V: \quad$ Vertical

S: $\quad$ Segmentation

I: $\quad$ Included

E: $\quad$ Ellipsoidal shape.

\section{Data Availability}

The data used to support the findings of this study are included within the article.

\section{Conflicts of Interest}

The authors declare that there are no conflicts of interest regarding the publication of this paper.

\section{Acknowledgments}

This work was partly supported by the Korea Institute of Energy Technology Evaluation and Planning (KETEP) grants funded by the Korean government (MOTIE) (no. 20191510301290, Development of Configuration Management Platform for Decommissioning of Nuclear Power Plants (no. 20203210100240), Development of a Platform for Optimization of Interdependences in Packaging-TransportDisposal of Radioactive Waste and History Management).

\section{References}

[1] International Atomic Energy Agency (IAEA), "Managing low radioactivity material from the decommissioning of nuclear facilities," Technical Reports Series No. 462, IAEA, Vienna, Austria, 2008.

[2] P. Petr, "Reactor vessel internals segmentation experience using mechanical cutting tools," Technical Engineering, vol. 10, no. 2, pp. 6-10, 2013.

[3] N. H. Lacy and Z. Studnicka, "One-piece reactor removal and disposal," in Proceedings of the Waste Management 2001, Tucson, AZ, USA, March 2001.

[4] F. Stefan, B. Joseph, S. Per, and J. K. Paul, "A proven method for performing steam dryer segmentation on BWR plants11479," in Proceedings of the Waste Management 2011, Phoenix, AZ, USA, March 2001.

[5] B. Joseph, P. Segerud, and M. Sanchez, "Best practice for preparing vessel internals segmentation projects," in Proceedings of the PREDEC 2016, Lyon, France, February 2016.

[6] Electric Power Research Institute (EPRI), "Reactor internals segmentation experience report, detailed experiences 1993-2006," Electric Power Research Institute (EPRI), Palo Alto, CA, USA, Tech No. 1015122, 2007.

[7] Electric Power Research Institute (EPRI), "Rancho Seco reactor vessel segmentation experience report," Electric Power Research Institute (EPRI), Palo Alto, CA, USA, Tech No. 1015501, 2008.

[8] Electric Power Research Institute (EPRI), "Maine Yankee decommissioning experience report, detailed experience 1997-2004," Electric Power Research Institute (EPRI), Palo Alto, CA, USA, Tech No. 1011734, 2005.

[9] Electric Power Research Institute (EPRI), "Decommissioning reactor pressure vessel internals segmentation," Electric Power Research Institute (EPRI), Palo Alto, CA, USA, Tech No. 1003029, 2001.

[10] H.-S. Park, S.-K. Kim, K.-W. Lee, C.-H. Jung, J.-H. Park, and S.-I. Jin, "The application of visualization and simulation in a dismantling process," Journal of Nuclear Science and Technology, vol. 44, no. 4, pp. 649-656, 2007.

[11] J. H. Lee, K. H. Kim, D. J. Hyun et al., "Derivation of segmentation method for highly activated reactor pressure vessel internal structure using 3-D digital model," in Proceedings of the Korean Nuclear Society Autumn Meeting, Jeju Island, Koreain Korean, Jeju Island, Korea, October 2013. 
[12] Y. Choi, S. C. Lee, S.-C. Lee, and C.-L. Kim, "Evaluation on radioactive waste disposal amount of Kori unit 1 reactor vessel considering cutting and packaging methods," Journal of Nuclear Fuel Cycle and Waste Technology, vol. 14, no. 2, pp. 123-134, 2016, in Korean.

[13] R. H. Perry, D. W. Green, and J. O. Maloney, Perry's Chemical Engineers' Handbook, McGraw-Hill, New York, NY, USA, Seventh edition, 1997.

[14] ALIO, "procurement specification [in Korean]," http://www. alio.go.kr/informationBidView.do?seq= 2204781Korea: Ministry of Economy and Finance.

[15] Rhinoceros: http://docs.mcneel.com/rhino/6/usersguide/enus/index.htmSeattle: Robert McNeel \& Associates.

[16] Advanced Reactors Information System (ARIS), "Reactor Pressure Vessel, Wall thickness of cylindrical shell [mm]," https://aris.iaea.org/sites/core.htmlAustria: International Atomic Energy Agency.

[17] Nuclear Regulatory Commission (NRC), Safety Evaluation Report Related to Steam Generator Repair at Turkey Point Plant Units 3 and 4, Nuclear Regulatory Commission (NRC), Rockville, MD, USA, 1980.

[18] The American Society of Mechanical Engineers (ASME), ASME B \& PV Code: Section VIII, Division 1, Design \& Fabrication of Pressure Vessels, The American Society of Mechanical Engineers (ASME), Park Avenue, NY, USA, 2020. 